Supporting Information

\title{
Unraveling the Role of Neutral Units for Single-Ion Conducting Polymer Electrolytes
}

Sheng Zhao, ${ }^{\S}$ Shenghan Song, "Yingqi Wang, Jong Keum, ${ }^{\dagger}$ Jiadeng Zhu, ${ }^{*}$ Yi He, " Alexei P. Sokolov ${ }^{*,+,+}$ and Peng-Fei Cao ${ }^{*+\hat{t}}$

$\S$ Department of Chemistry, University of Tennessee, Knoxville, Tennessee 37996, USA

$\dagger$ Center for Nanophase Materials Science and Neutron Scattering Division, $₫$ Chemical Sciences Division, Oak Ridge National Laboratory, Oak Ridge, Tennessee 37830, USA.

" Chemistry Department of Chemistry and Chemical Biology, The University of New Mexico, Albuquerque, New Mexico 87131, USA

\section{Corresponding Authors}

*E-mail: sokolov@utk.edu (A.S.)

*E-mail: caop@ornl.gov (P.C.) 


\section{EXPERIMENTAL SECTION}

Materials Poly(ethylene glycol) methyl ether methacrylate (PEGMEMA, 500g/mol), 2ethylhexyl acrylate (EHA), acryloyl chloride, 4-(hydroxymethyl)-1,3-dioxolan-2-one (glycerol carbonate, GC), lithium perchlorate $\left(\mathrm{LiClO}_{4}\right)$ and magnesium sulfate anhydrous $\left(\mathrm{MgSO}_{4}\right)$ were purchased from Sigma Aldrich. Monomethacryloxylpropyl terminated polydimethylsiloxane, asymmetric (PDMSmMA, 900g/mol) was purchased from Gelest. Carboxylic acid terminated RAFT agent 2-[(butylsulfanyl) carbothioyl] sulfanyl propanoic acid (RAFT-CTA) was synthesized according to the previous paper. ${ }^{1,2}$ Azobisisobutyronitrile (AIBN) was recrystallized from methanol before use. The monomers, i.e. PEGMEMA, EHA, PDMSmMA and GCA, were passed through a column with alternative inhibitor remover and aluminum oxide to remove inhibitor before polymerization.

Synthesis of lithium (4-styrenesulfonyl)(trifluoromethanesulfonyl)imide (STFLi) Due to the high sensitivity of STFLi towards the moisture, the STFK was synthesized instead based on literature with minor change. ${ }^{3,4}$ The successful synthesis of monomer was verified by ${ }^{1} \mathrm{H}$ NMR spectrum as shown in Figure S1(A). STFK monomer was dried under vacuum transferring to the glovebox. The equal mole ratio of $\mathrm{LiClO}_{4}$ and STFK were dissolved in dry tetrahydrofuran (THF) and stirred overnight for completing ion exchange. The filtered THF solution of STFLi was used for the following reactions.

STFK ${ }^{1} \mathrm{H}$ NMR (400 MHz, DMSO-d $) \delta 7.78$ - $7.65(\mathrm{~m}, 2 \mathrm{H}), 7.58(\mathrm{dd}, 2 \mathrm{H}), 6.90$ - $6.70(\mathrm{~m}$, 1H), $5.95(\mathrm{dd}, 1 \mathrm{H}), 5.38(\mathrm{dd}, 1 \mathrm{H})$. 
Synthesis of (2-Oxo-1,3-dioxolan-4-yl) acrylate or glycerin carbonate acrylate (GCA) $2.5 \mathrm{~g}$ GC (21.2 mmol) and $2.6 \mathrm{~mL}$ acryloyl chloride $(31.8 \mathrm{mmol})$ were separately dissolved in $20 \mathrm{~mL}$ and $10 \mathrm{~mL}$ anhydrous chloroform, respectively. The solution with GC was loaded in a two-neck round bottom flask with one of the necks connected with a dropping funnel and the other one sealed. The system has been degassed for $15 \mathrm{~min}$ before the addition of acryloyl chloride solution through the dropping funnel by. The reaction was conducted for $6 \mathrm{~h}$ under Argon condition at $0^{\circ} \mathrm{C}$. The resulted solution was washed with $10 \mathrm{~mL}$ of $1 \mathrm{M} \mathrm{NaHCO}_{3}$ aqueous solution to remove extra acryloyl chloride and then extracted by chloroform for three times. The organic layer was dried by the anhydrous $\mathrm{MgSO}_{4}$, and the monomer was collected after removing chloroform by rotary evaporation at room temperature. The successful synthesis of monomer was verified by ${ }^{1} \mathrm{H}$ NMR spectrum (Figure S5(A)).

GCA ${ }^{1} \mathrm{H}$ NMR (400 MHz, Chloroform- $d$ ) $\delta 6.46(\mathrm{dd}, 1 \mathrm{H}), 6.15(\mathrm{dd}, 1 \mathrm{H}), 5.94(\mathrm{dd}, 1 \mathrm{H}), 5.03-$ $4.95(\mathrm{~m}, 1 \mathrm{H}), 4.60(\mathrm{t}, 1 \mathrm{H}), 4.45(\mathrm{dd}, 1 \mathrm{H}), 4.38-4.32(\mathrm{~m}, 2 \mathrm{H})$.

\section{Synthesis of 2-[(1,1-Dioxidotetrahydro-3-thienyl)oxy]ethanol acrylate (DTTA)}

A similar procedure with GCA was applied and the mass of 2-[(1,1-Dioxidotetrahydro-3thienyl)oxy]ethanol (DTT) was adjusted based on the molecular weight $(180.22 \mathrm{~g} / \mathrm{mol})$. The successful synthesis of monomer was verified by ${ }^{1} \mathrm{H}$ NMR spectrum (Figure S7(A)).

DTTA ${ }^{1} \mathrm{H}$ NMR (400 MHz, Chloroform-d) $6.38(\mathrm{dd}, 1 \mathrm{H}), 6.23-5.98(\mathrm{~m}, 1 \mathrm{H}), 5.83(\mathrm{~d}, 1 \mathrm{H})$, $4.45-4.05(\mathrm{~m}, 3 \mathrm{H}), 3.67(\mathrm{t}, 2 \mathrm{H}), 3.27-2.95(\mathrm{~m}, 4 \mathrm{H}), 2.34(\mathrm{t}, 2 \mathrm{H})$.

\section{Synthesis of 3-(Methylsulfonyl)-1-ethanol acrylate (MSEA)}


A same procedure with GCA was applied and the mass of 3-(Methylsulfonyl)-1-ethanol (MSE) was adjusted based on the molecular weight $(124.16 \mathrm{~g} / \mathrm{mol})$. The successful synthesis of monomer was verified by ${ }^{1} \mathrm{H}$ NMR spectrum (Figure S7(B)).

MSEA ${ }^{1} \mathrm{H}$ NMR (400 MHz, Chloroform-d) 6.36 (dd, 1H), 6.05 (ddd, 1H), $5.91-5.80$ (m, 1H), $4.51(\mathrm{dt}, 2 \mathrm{H}), 3.32(\mathrm{q}, 2 \mathrm{H}), 2.94-2.84(\mathrm{~m}, 3 \mathrm{H})$

General procedure for synthesizing homopolymers In a typical synthesis process of poly(PEGMEMA), $11 \mathrm{mg}$ RAFT-CTA (0.05 mmol), $988 \mathrm{mg}$ PEGMEMA (2.0 mmol), 2 mg AIBN (0.05 mmol) were added into a round bottom flask equipped with a stir bar and dissolved by $7 \mathrm{~mL}$ dry THF. After purged with Argon for $1 \mathrm{hr}$ at room temperature, the flask was sealed and the reaction was performed at $65^{\circ} \mathrm{C}$ with magnetic stirring for $24 \mathrm{~h}$. The mixture was purified by dialysis against THF. The sample was dried in a vacuum oven at $110^{\circ} \mathrm{C}$ for two days to fully remove solvents before further characterizations. The homopolymers with comparable degree of polymerization $\left(\mathrm{DP}_{\mathrm{n}}\right)$ for different monomers were synthesized in the similar manner. (See Figure S1(B), S2-4(A), S5(B) and S8 for ${ }^{1} \mathrm{H}$ NMR spectra.)

Poly(STFLi) ${ }^{1} \mathrm{H}$ NMR (400 MHz, DMSO-d $\left.d_{6}\right) \delta 7.47$ (s, 2H), 6.51 (s, 2H), 2.50 (d, 1H), $2.11-0.49$ $(\mathrm{m}, 2 \mathrm{H})$.

Poly(PEGMEMA) ${ }^{1} \mathrm{H}$ NMR (400 MHz, Chloroform- $d$ ) $\delta 4.05$ (s, 2H), 3.62 (d, 33H), 3.52 (dd, 2H), $3.35(\mathrm{~s}, 3 \mathrm{H}), 1.75(\mathrm{~s}, 2 \mathrm{H}), 1.08-0.70(\mathrm{~m}, 3 \mathrm{H})$.

Poly(EHA) ${ }^{1} \mathrm{H}$ NMR (400 MHz, Chloroform- $d$ ) $\delta 4.16-3.76(\mathrm{~m}, 2 \mathrm{H}), 2.50-2.18(\mathrm{~m}, 1 \mathrm{H}), 2.01-$ $1.73(\mathrm{~m}, 1 \mathrm{H}), 1.75-1.51(\mathrm{~m}, 2 \mathrm{H}), 1.47-1.16(\mathrm{~m}, 8 \mathrm{H}), 0.88(\mathrm{q}, 6 \mathrm{H})$.

Poly(PDMSmMA) ${ }^{1} \mathrm{H}$ NMR (400 MHz, Chloroform- $d$ ) $\delta 3.88(\mathrm{~s}, 2 \mathrm{H}), 1.88(\mathrm{~s}, 2 \mathrm{H}), 1.46$ - 1.20 (m, $6 \mathrm{H}), 0.95-0.73(\mathrm{~m}, 6 \mathrm{H}), 0.52(\mathrm{~d}, 4 \mathrm{H}), 0.30--0.37(\mathrm{~m}, 100 \mathrm{H})$. 
Poly(GCA) ${ }^{1} \mathrm{H}$ NMR (400 MHz, DMSO-d $) \delta 5.02(\mathrm{~s}, 1 \mathrm{H}), 4.59$ (t, 1H), $4.43-4.02$ (m, 3H), 2.33 $(\mathrm{s}, 1 \mathrm{H}), 2.02-1.39(\mathrm{~m}, 2 \mathrm{H})$.

Poly(DTTA) ${ }^{1}$ H NMR (400 MHz, DMSO-d6) $\delta 4.35$ (s, 1H), 4.13 (s, 2H), 3.62 (s, 2H), 3.35 (s, 1H), $3.12(\mathrm{~s}, 3 \mathrm{H}), 2.27(\mathrm{~s}, 3 \mathrm{H}), 1.62(\mathrm{~s}, 2 \mathrm{H})$.

Poly(MSEA) ${ }^{1} \mathrm{H}$ NMR (400 MHz, DMSO-d6) $\delta 4.37$ (s, 2H), 3.49 (s, 2H), 3.04 (s, 3H), 2.32 (s, 1H), $1.64(\mathrm{~s}, 2 \mathrm{H})$.

General procedure for RAFT synthesizing SICPEs In a typical one pot synthesis, $26 \mathrm{mg}$ RAFT-CTA (0.11 mmol), $1.2 \mathrm{~g}$ PEGMEMA (2.4 mmol) and $3.6 \mathrm{mg}$ AIBN (0.02 mmol) were dried under vacuum before transferring to glovebox. The THF solution containing $772 \mathrm{mg}$ STFLi (2.4 mmol) was added into the round bottom flask containing the above chemicals, followed by addition of another 10mL of dry THF. After purged with Argon for $1 \mathrm{hr}$ at room temperature, the flask was sealed and the reaction was performed at $65^{\circ} \mathrm{C}$ with magnetic stirring for $24 \mathrm{~h}$. The product was purified by dialysis against THF and was dried under vacuum at $110^{\circ} \mathrm{C}$ for two days before the characterizations. (See Figure 1(A), S2-4(B), S6 and S9 for ${ }^{1} \mathrm{H}$ NMR spectra.)

\section{Characterization}

${ }^{1} \mathrm{H}$ Nuclear magnetic resonance $\left({ }^{1} \mathrm{H}\right.$ NMR) measurements were performed on a Bruker Advance III $400 \mathrm{MHz}$ NMR Spectrometer with d-Chloroform, d-THF or d-DMSO as solvents. Fourier transform infrared spectroscopy (FT-IR) was measured on a Nicolet iS50 FT-IR spectrometer equipped with a deuterated triglycine sulfate (DTGS) detector. The spectra were obtained with 64 scans and averaged with signals. 
The thermal stability of samples was measured by thermogravimetric analysis (TGA) on TA instrument TGA Q-50 under $\mathrm{N}_{2}$ with a heating rate of $20^{\circ} \mathrm{C} / \mathrm{min}$ from room temperature to $800^{\circ} \mathrm{C}$. Differential scanning calorimetry (DSC) measurements were performed under Ar atmosphere using a TA instrument DSC 2500. The samples were placed into aluminum hermetic pans, dried in the vacuum oven at $100{ }^{\circ} \mathrm{C}$ overnight and sealed before the test. The samples were equilibrated at $120^{\circ} \mathrm{C}$ for $30 \mathrm{~min}$ to remove the thermal history. The cooling and heating cycles were perfomed with a rate of $3{ }^{\circ} \mathrm{C} / \mathrm{min}$ in the range of $-90{ }^{\circ} \mathrm{C}$ to $250{ }^{\circ} \mathrm{C}$ (from $-140{ }^{\circ} \mathrm{C}$ to $200{ }^{\circ} \mathrm{C}$ for poly(PDMSmMA $\left.\left.{ }_{17} / \mathrm{STFLi}_{23}\right)\right)$. $\mathrm{T}_{\mathrm{g}}$ was estimated as the midpoint of the transition process in the final heating cycle.

Small angle X-ray scattering (SAXS) measurements were performed on an Anton Paar SAXSess mc2 at the Center for Nanophase Materials Sciences (CNMS) of the Oak Ridge National Laboratory. The SAXS images were collected with a CCD detector (PI-SCX, Roper) at a pixel resolution of $2084 \times 2084$ and pixel dimensions of $24 \times 24 \mu \mathrm{m}^{2}$. For the measurements, X-rays were generated at $40 \mathrm{kV} / 50 \mathrm{~mA}$ at a beam wavelength of $\lambda=1.541 \AA$ ( $\mathrm{Cu} \mathrm{K \alpha}$ radiation), while the data was for $20 \mathrm{~min}$.

Small-amplitude oscillatory shear (SAOS) measurements were performed on an AR2000ex rheometer. The experiments were performed between two $4 \mathrm{~mm}$ parallel steel plates. The temperature was controlled by a system using nitrogen as the gas source. All the samples were loaded between the plates, equilibrated at $60^{\circ} \mathrm{C}$ for 30 mins before the measurement, and measured at different temperatures with the angular frequency sweep from 100 to $0.1 \mathrm{rad} / \mathrm{s}$. 
Broadband Dielectric Spectroscopy (BDS) was performed in the frequency range of $10^{-1} \sim 10^{-6}$ $\mathrm{Hz}$ by using a Novocontrol Concept 80 system. The samples were sandwiched with two gold plated electrodes separated by a Teflon spacer. All the samples were dried in the vacuum oven at $120^{\circ} \mathrm{C}$ overnight prior to the measurement. During the measurements, samples were equilibrated at 120 ${ }^{\circ} \mathrm{C}\left(170{ }^{\circ} \mathrm{C}\right.$ for $\operatorname{poly}\left(\mathrm{GCA}_{\mathrm{x}} / \mathrm{STFLi}_{\mathrm{y}}\right)$, poly(DTTA $\left.20 / \mathrm{STFLi}_{20}\right)$, poly(MSEA $\left.19 / \mathrm{STFLi} 21\right)$ and poly(PDMSmMA $\left.{ }_{17} / \mathrm{STFLi}_{23}\right)$ ) for $30 \mathrm{~min}$, then proceeded to $-60{ }^{\circ} \mathrm{C}$ and went back to the same high temperature under $\mathrm{N}_{2}$. The repeatable data at high temperature for both cycles suggest the absence of solvent or no degradation happened. Each temperature was equilibrated for $10 \mathrm{~min}$ before the measurements.

To test the electrochemical stability, the stainless steel/lithium cell composed of PC doped SICPEs was measured by linear sweep voltammetry (LSV) with a sweep rate of $5 \mathrm{mV} / \mathrm{s}$. Since the synthetic SICPEs are either highly viscous fluid or powder at ambient temperature, 10wt\% of selected SICPEs were separately dissolved in propylene carbonate (PC) for coin cell preparation. 


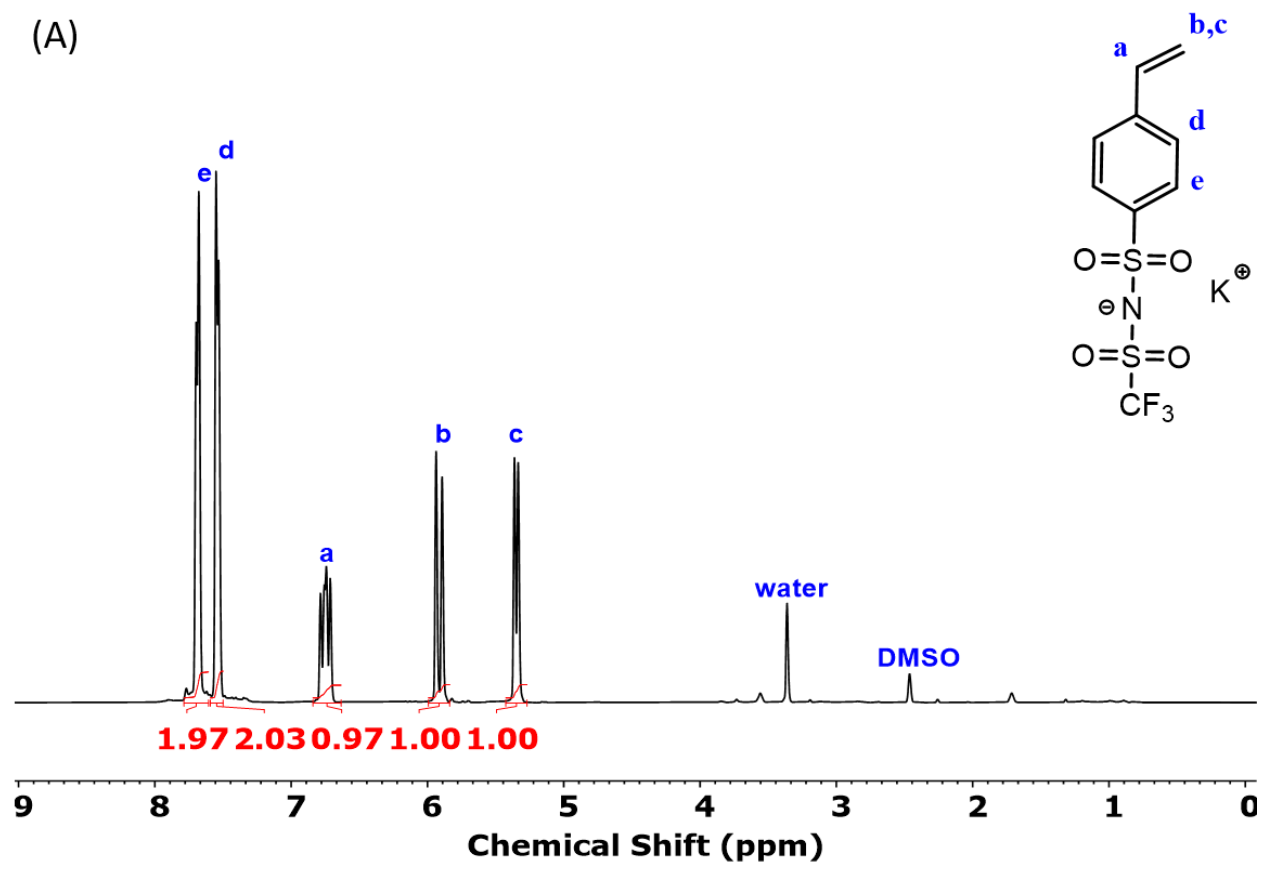

(B)
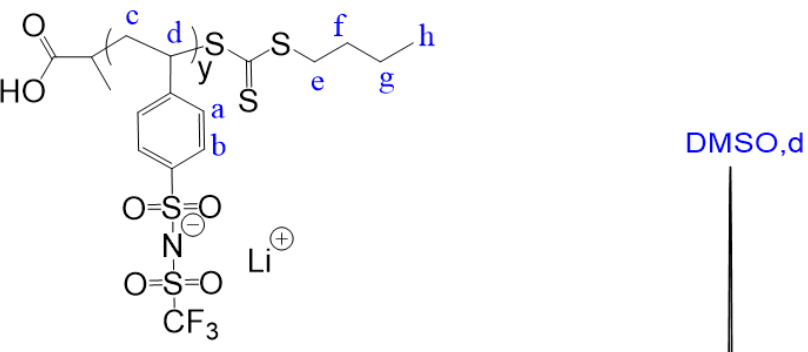

Figure S1. ${ }^{1} \mathrm{H}$ NMR spectra of (A) STFK and (B) poly(STFLi) in d-DMSO. 


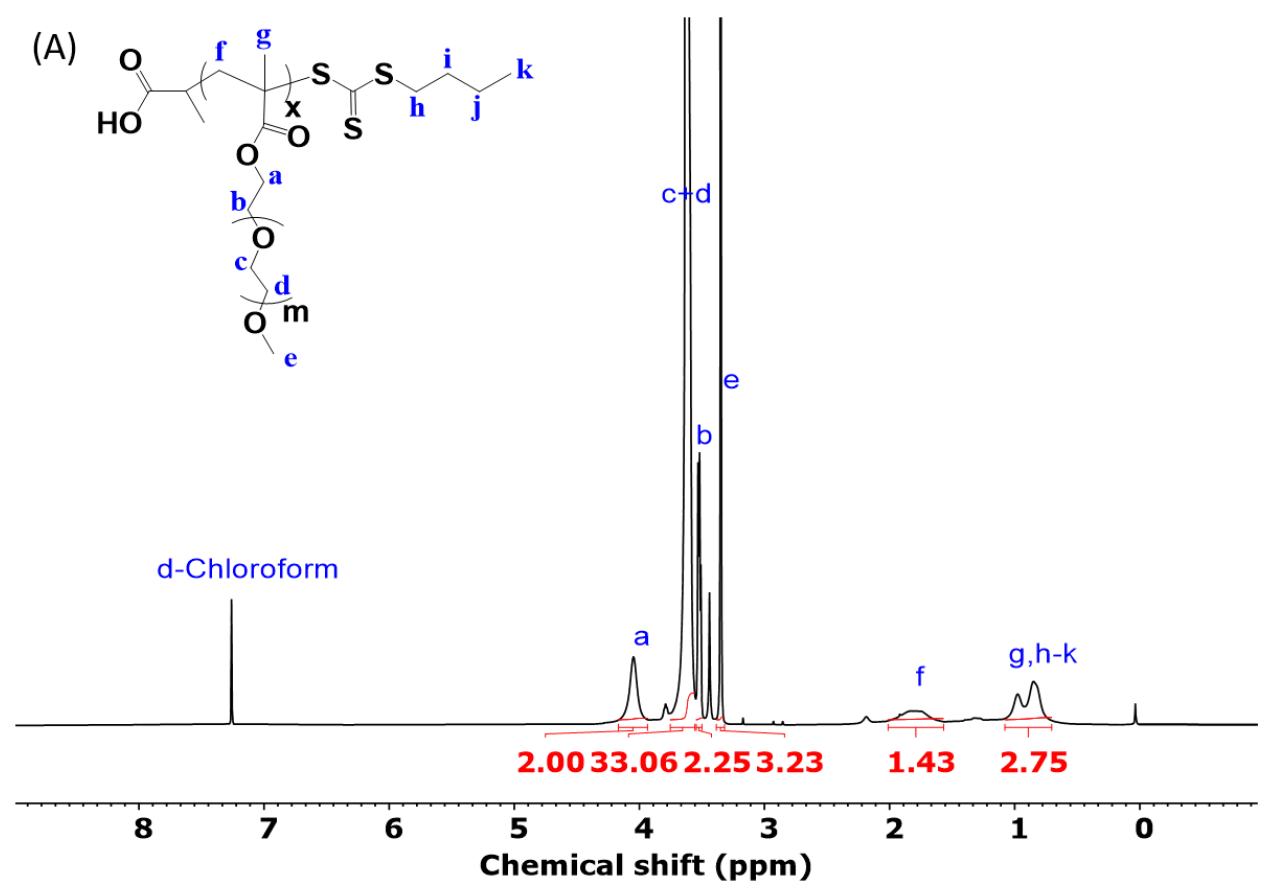

(B)
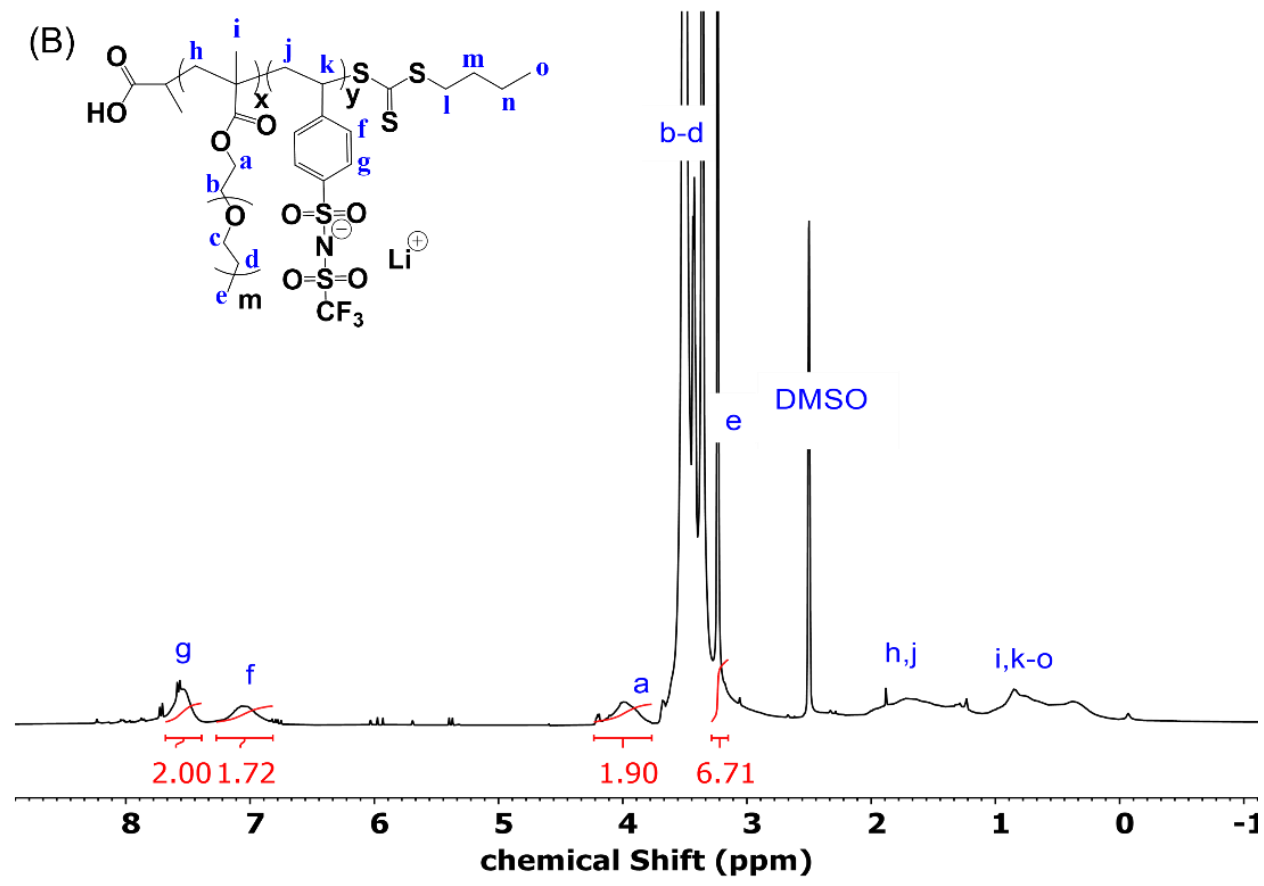

Figure S2. ${ }^{1} \mathrm{H}$ NMR spectra of (A) poly(PEGMEMA) in d-chloroform and (B) poly $\left(\right.$ PEGMEMA $\left._{25} / \mathrm{STFLi}_{15}\right)\left(\mathrm{STFLi}=40 /\left(1+\frac{\frac{a+e}{5}}{\frac{f+g}{4}}\right)=15\right.$, PEGMEMA $\left.=25\right)$ in $\mathrm{d}-\mathrm{DMSO}$. 

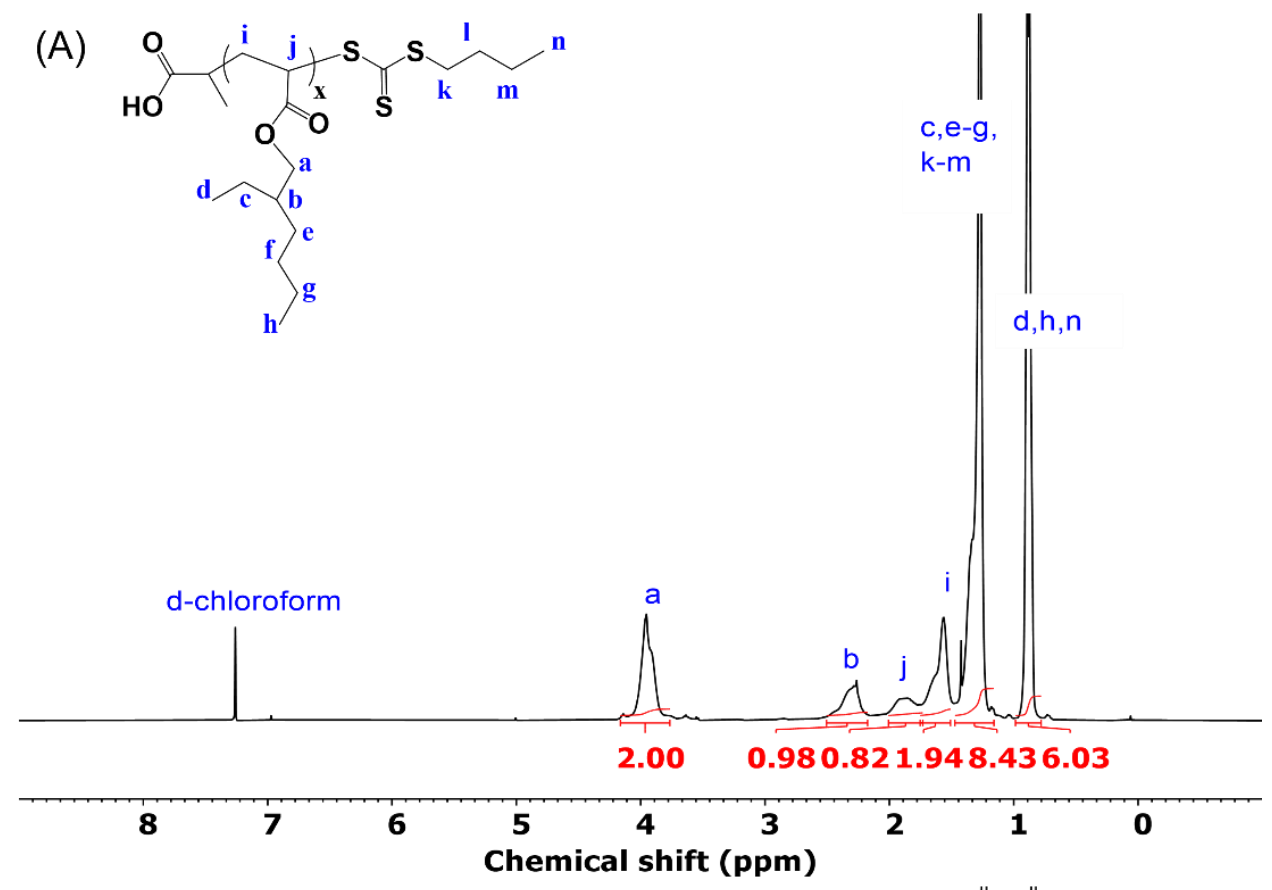

(B)
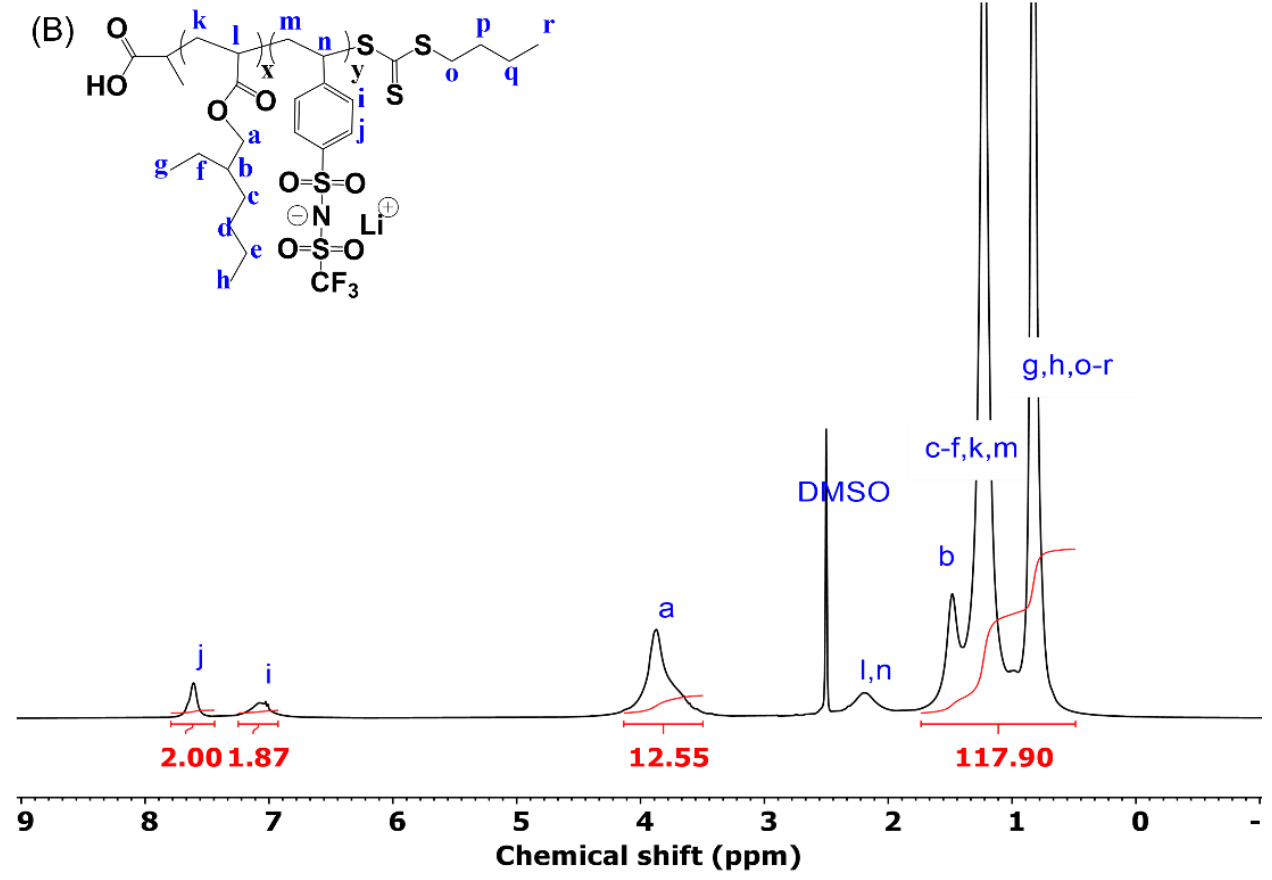

Figure S3. ${ }^{1} \mathrm{H}$ NMR spectra of (A) poly(EHA) in d-chloroform and (B) poly(EHA ( $\left._{34} / \mathrm{STFLi}_{6}\right)$ $\left(\mathrm{STFLi}=40 /\left(1+\frac{\left(\frac{a+b+c+d+e+f+g+h+k+m}{2+1+2 \times 3+3 \times 2+2 \times 2}\right)}{\frac{i+j}{4}}\right)=6, \mathrm{EHA}=34\right)$ in d-DMSO. 

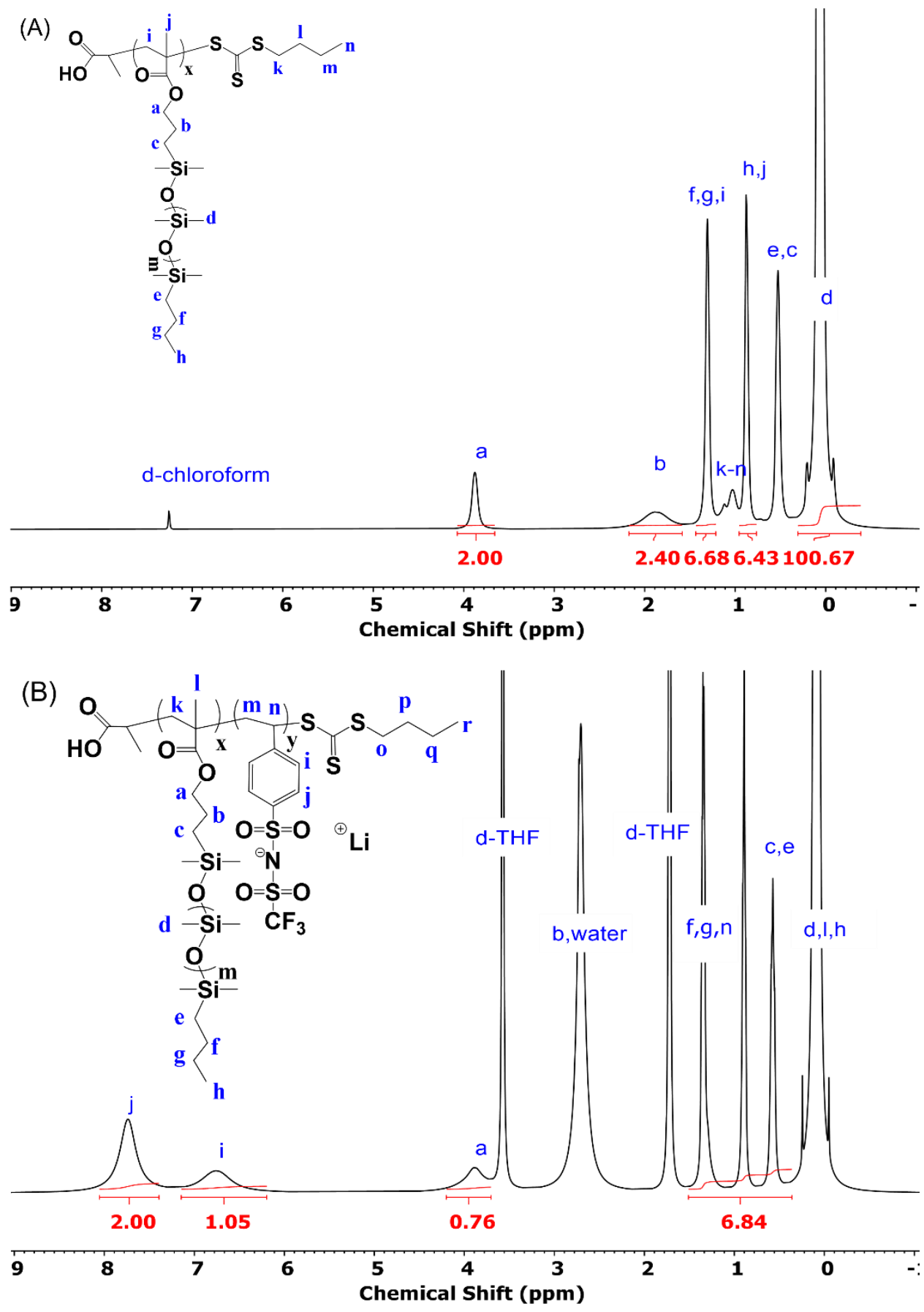

Figure S4. ${ }^{1} \mathrm{H}$ NMR spectra of (A) poly(PDMSmMA) in d-chloroform and (B) $\operatorname{poly}\left(\mathrm{PDMSmMA}_{17} / \mathrm{STFLi}_{23}\right)\left(\mathrm{STFLi}=40 /\left(1+\frac{\frac{a+c+e+f+g+k+m+n}{5}}{\frac{j+i}{4}}\right)=23, \mathrm{PDMSmMA}=17\right)$ in $\mathrm{d}-$ tetrahydrofuran. 
(A)

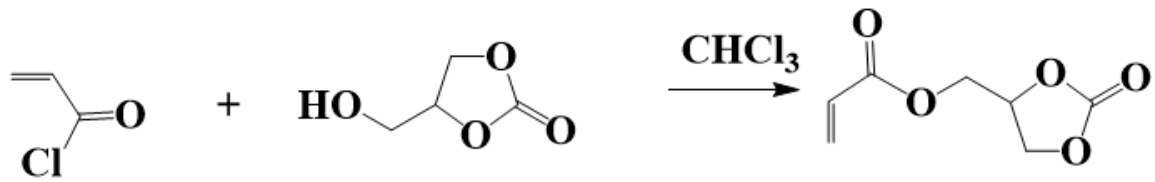

glycerin carbonate

(B)

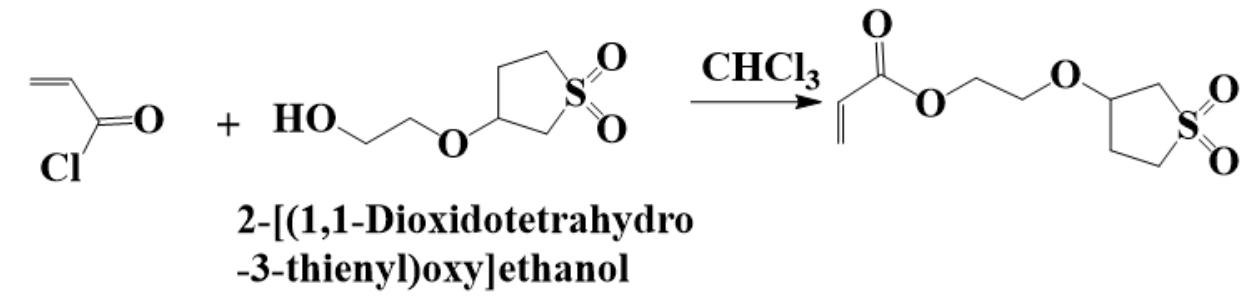

(C)

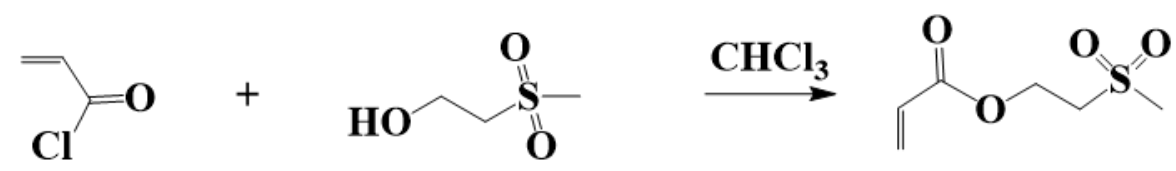

\section{3-(Methylsulfonyl)-1-ethanol}

Scheme S1. Synthesis of monomer (A) (2-Oxo-1,3-dioxolan-4-yl) acrylate (GCA), (B) 2-[(1,1Dioxidotetrahydro-3-thienyl)oxy]ethanol acrylate (DTTA), and (C) 3-(Methylsulfonyl)-1-ethanol acrylate (MSEA). 

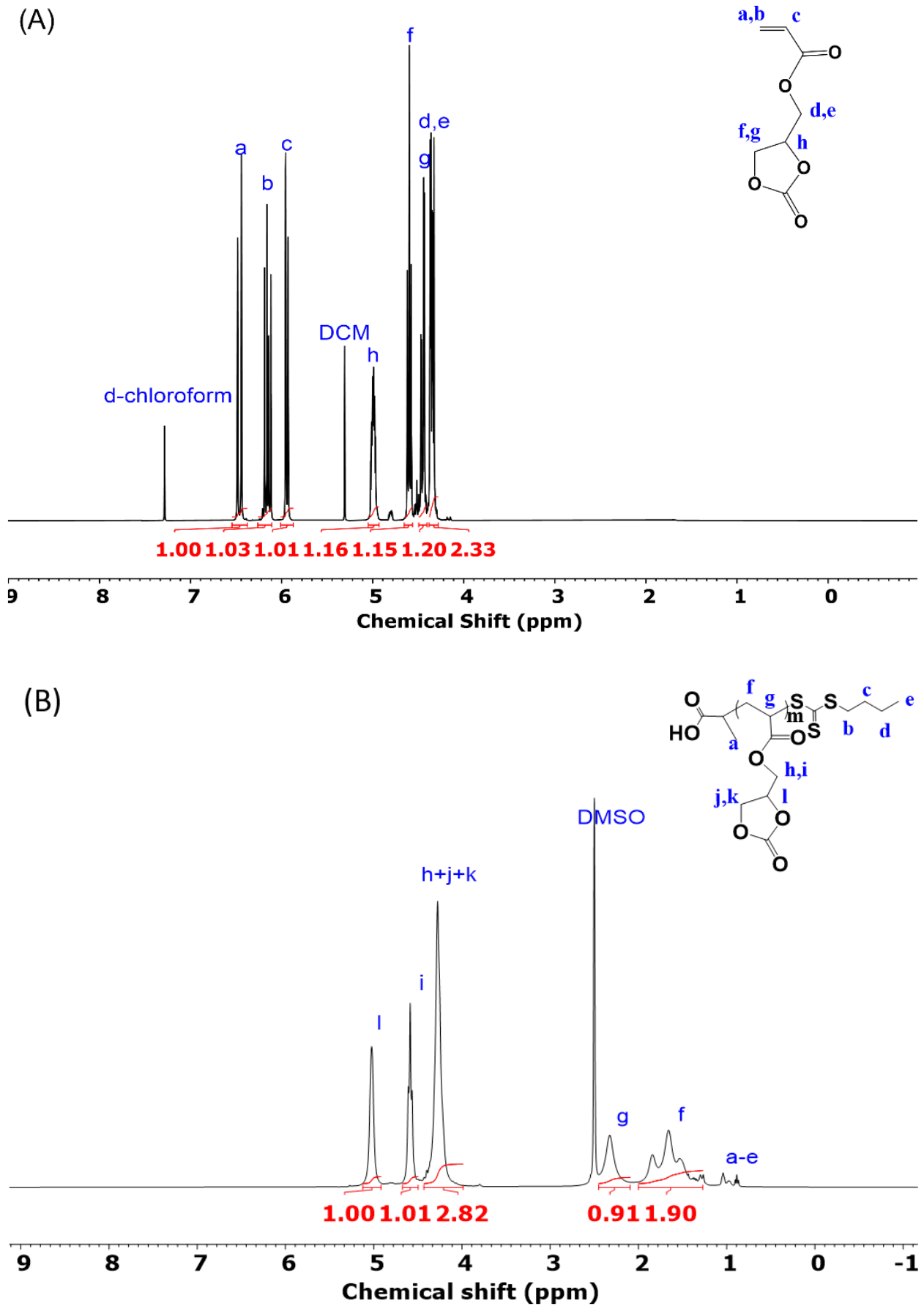

Figure S5. ${ }^{1} \mathrm{H}$ NMR of (A) GCA in d-chloroform and (B) poly(GCA) in d-DMSO. 

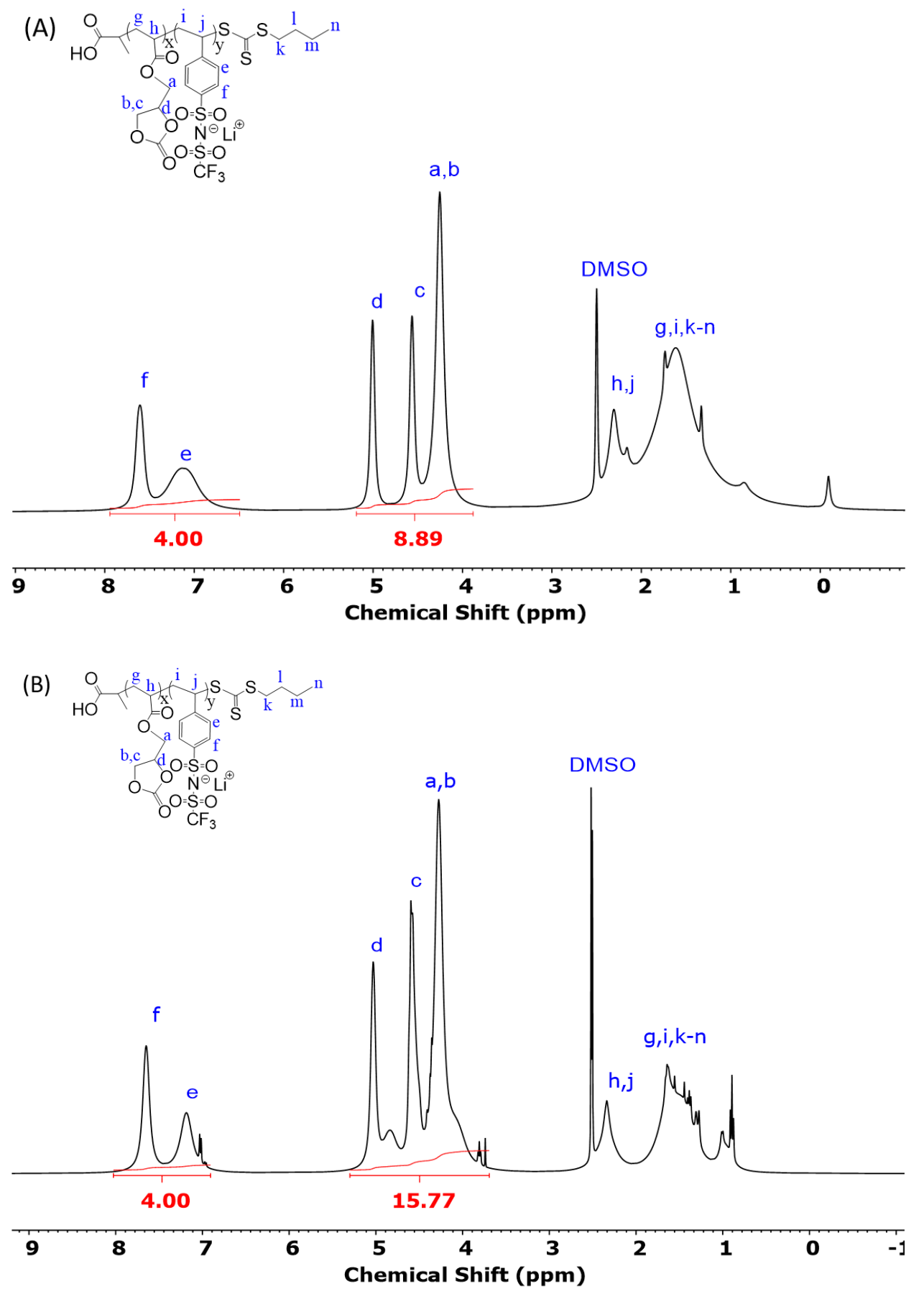

Figure S6. ${ }^{1} \mathrm{H}$ NMR spectra of (A) poly $\left(\mathrm{GCA}_{25} / \mathrm{STFLi}_{15}\right)\left(\mathrm{STFLi}=40 /\left(1+\frac{(a+b+c+d+e) / 5}{(e+f) / 4}\right)\right.$

$=15, \mathrm{GCA}=25)$ and $(\mathrm{B}) \operatorname{poly}\left(\mathrm{GCA}_{30} / \mathrm{STFLi}_{10}\right)\left(\mathrm{STFLi}=40 /\left(1+\frac{(a+b+c+d+e) / 5}{(e+f) / 4}\right)=10, \mathrm{GCA}=30\right)$ in d-DMSO. 

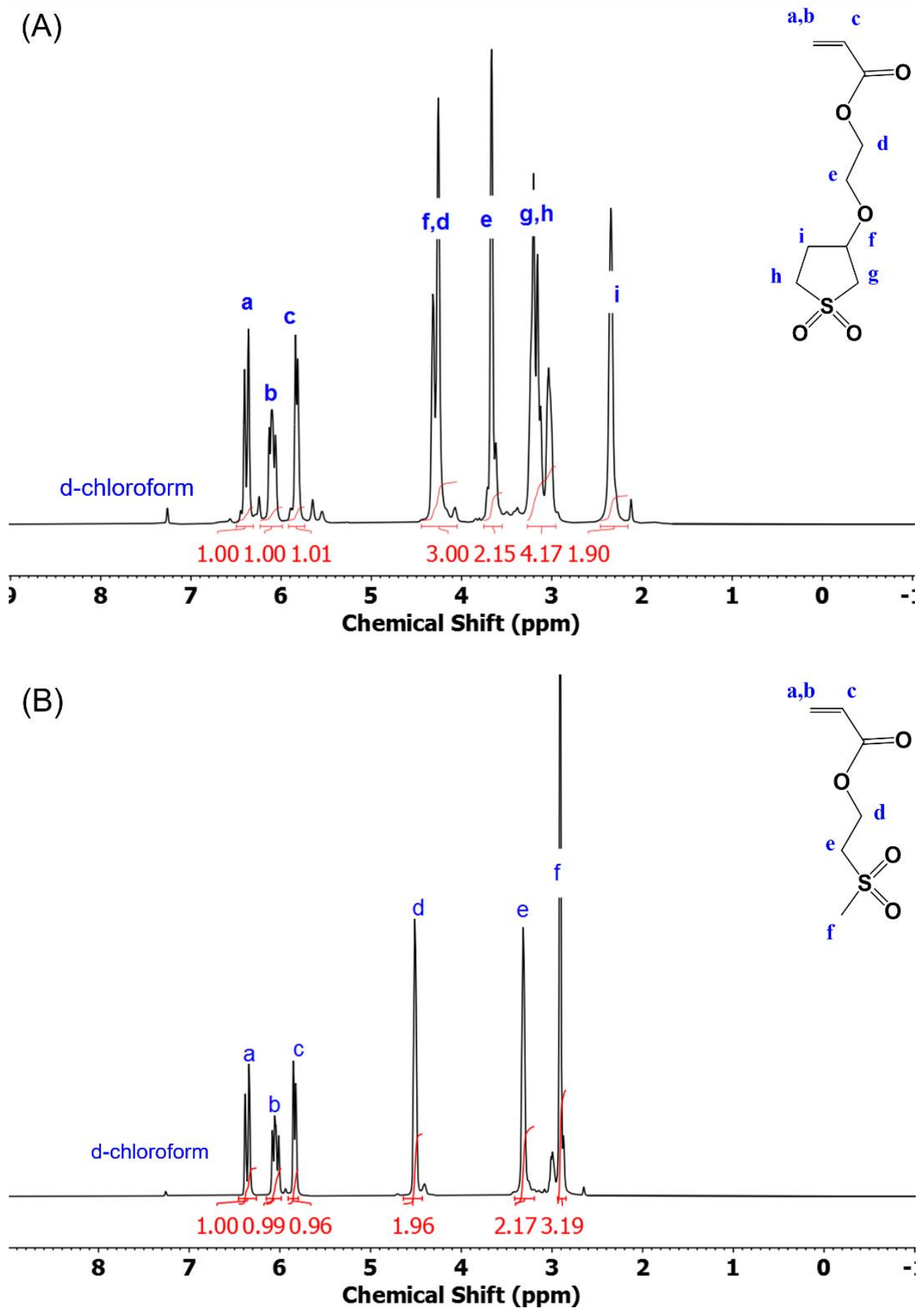

Figure S7. ${ }^{1} \mathrm{H}$ NMR of (A) DTTA and (B) MSEA in d-chloroform. 


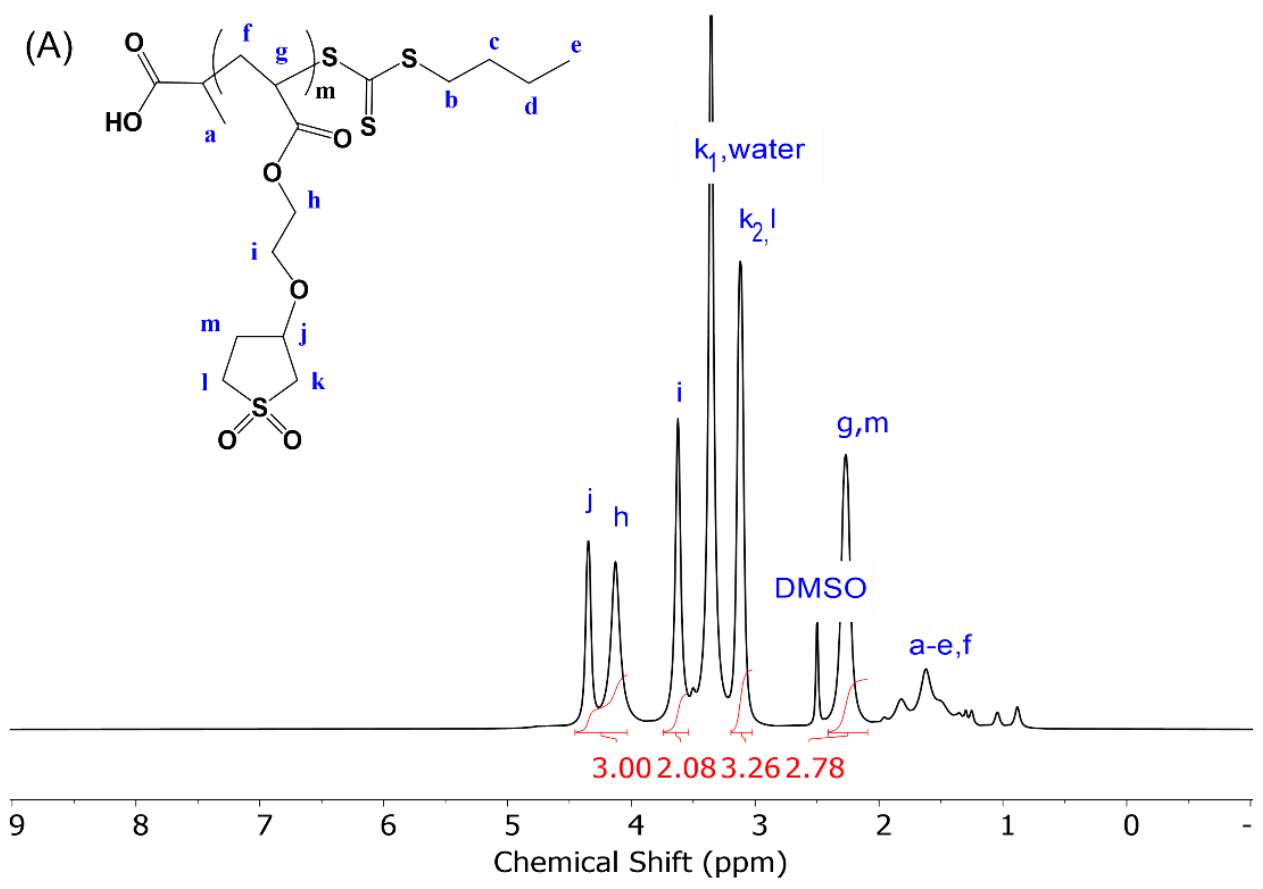

(B)

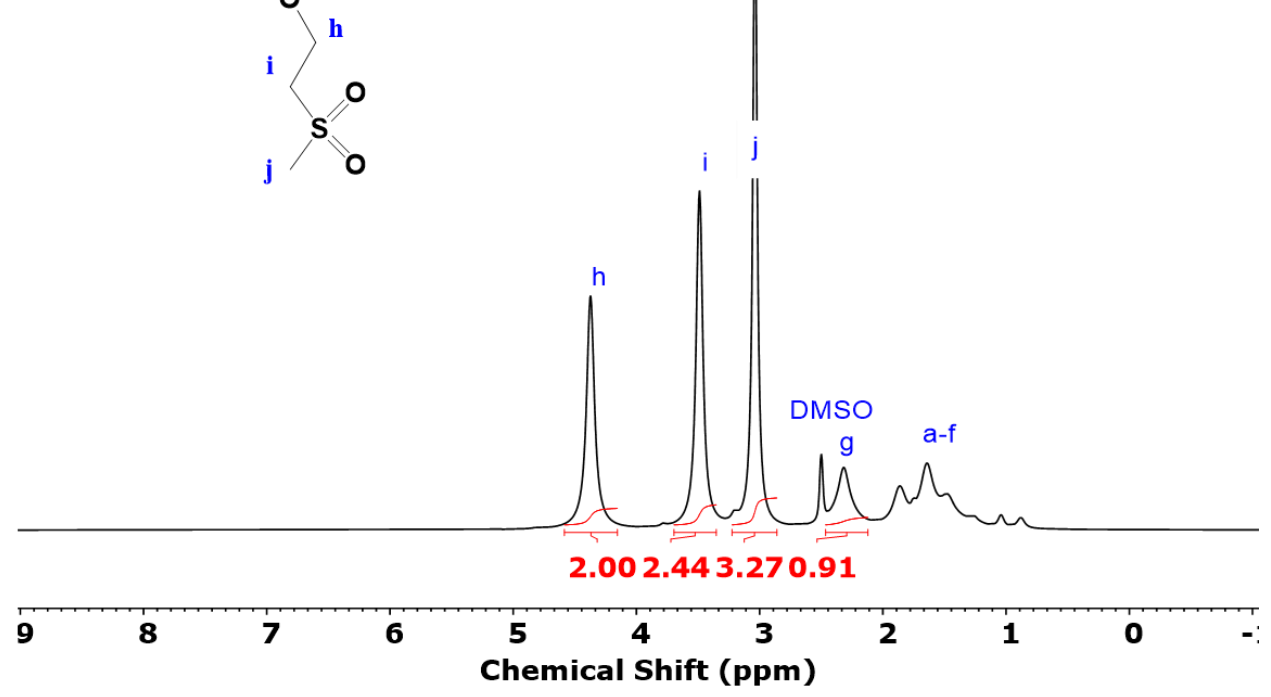

Figure S8. ${ }^{1} \mathrm{H}$ NMR of (A) poly(DTTA) and (B) poly(MSEA) in d-DMSO. 


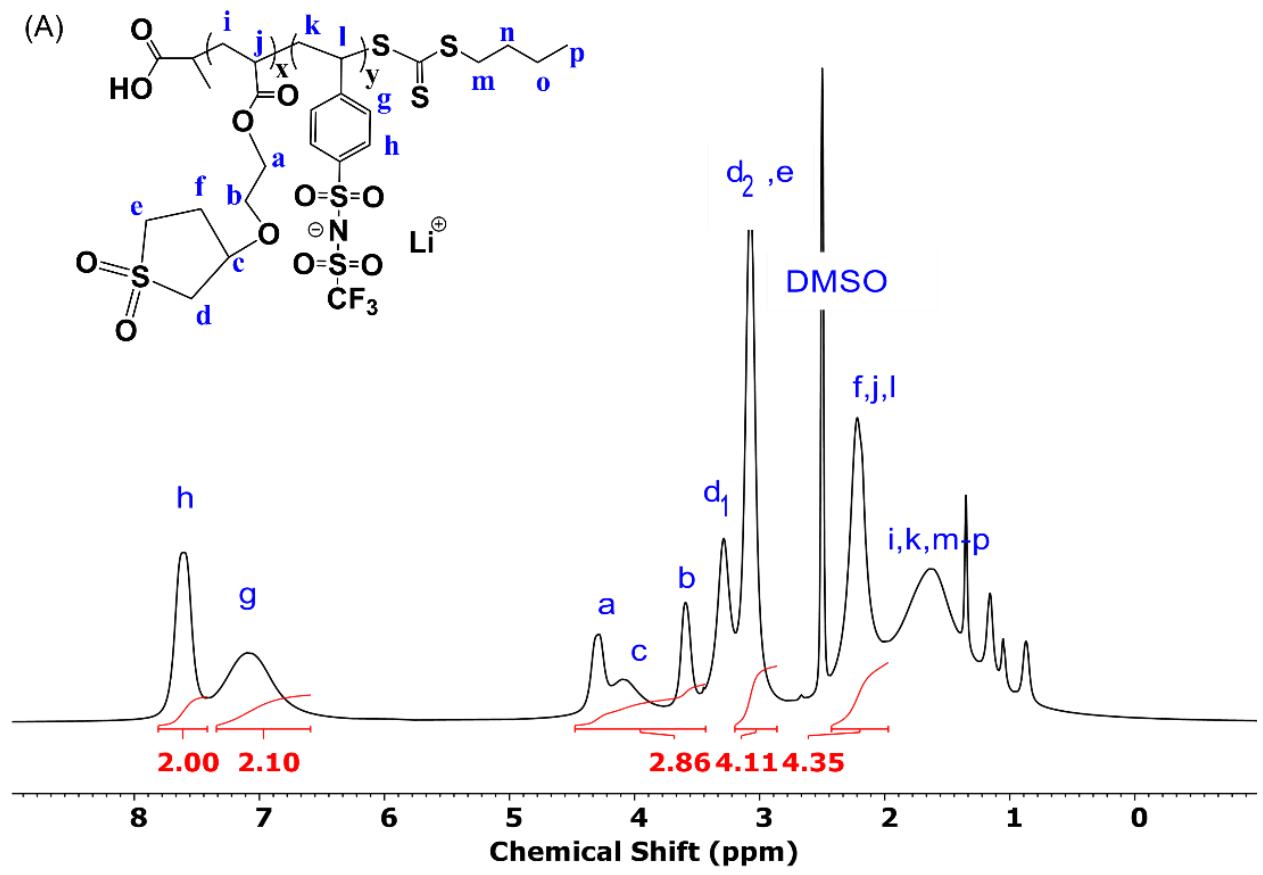

(B)
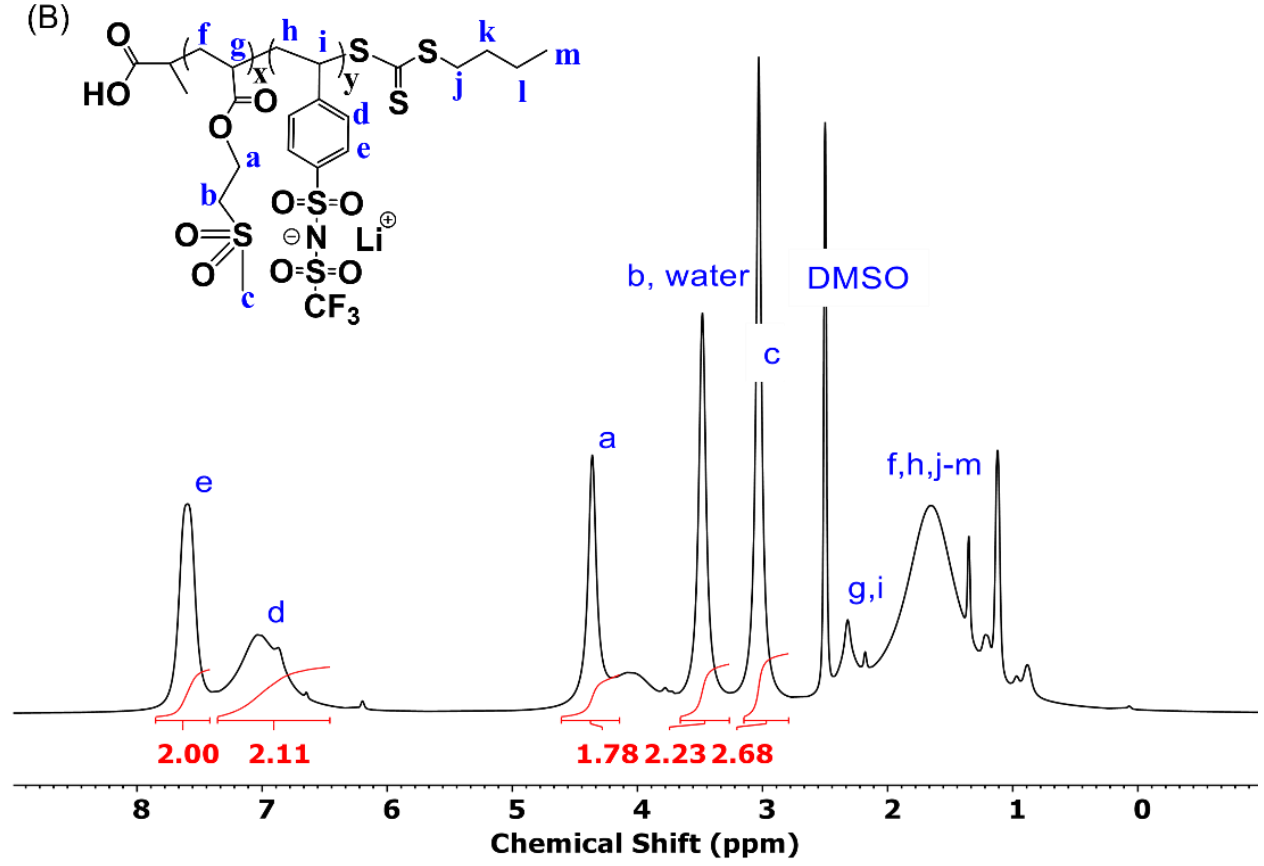

Figure S9. ${ }^{1} \mathrm{H}$ NMR of $(\mathrm{A})$ poly $\left(\mathrm{DTTA}_{20} / \mathrm{STFLi}_{20}\right)\left(\mathrm{STFLi}=40 /\left(1+\frac{(a+b+c+d+e+f+j+l) / 13}{(h+g) / 4}\right)=20\right.$, $\left.\mathrm{DTTA}^{20}\right)$ and $(\mathrm{B}) \operatorname{poly}\left(\mathrm{MSEA}_{21} / \mathrm{STFLi}_{19}\right)\left(\mathrm{STFLi}=40 /\left(1+\frac{(a+c) / 5}{(e+d) / 4}\right)=21, \mathrm{MSEA}=19\right)$ in $\mathrm{d}-$ DMSO. 
(A)

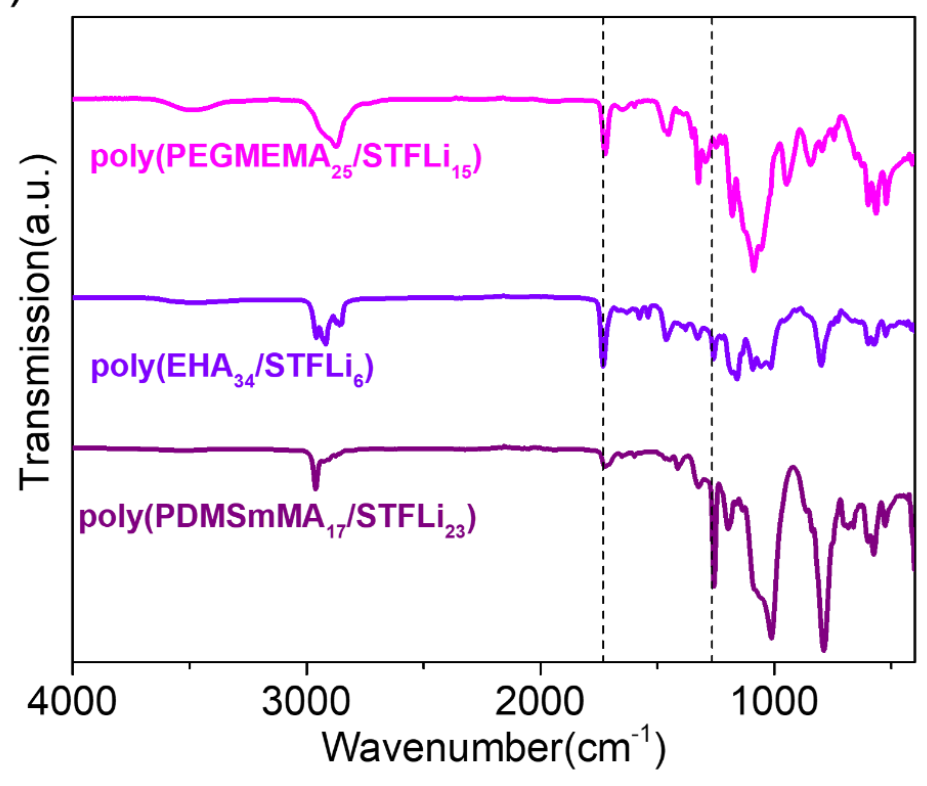

(B)

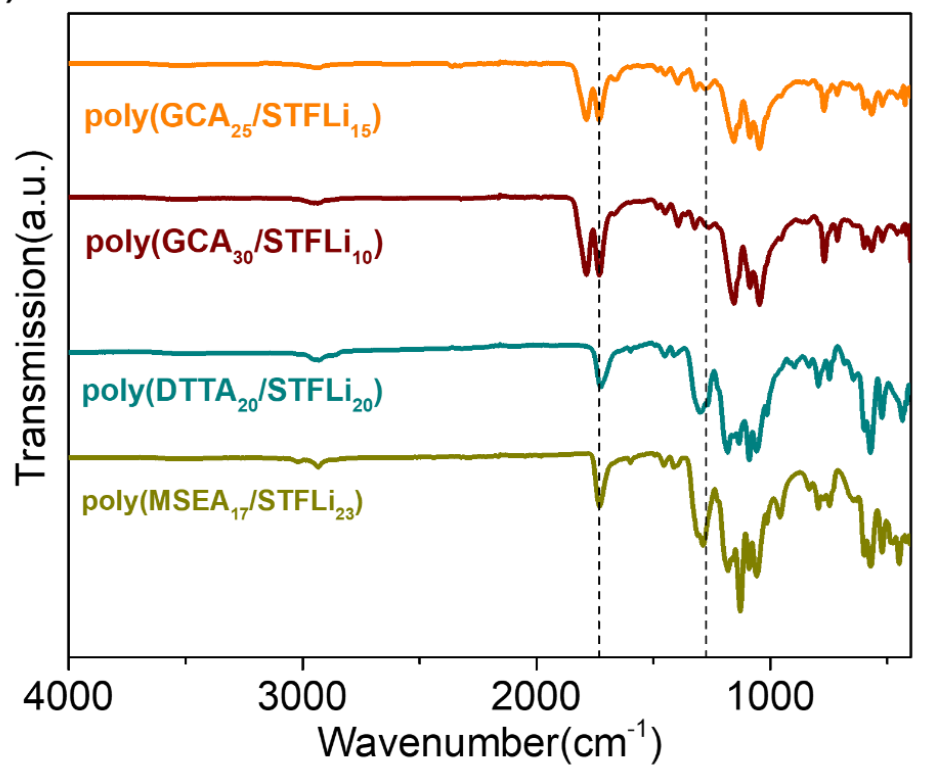

Figure S10. FT-IR spectra of (A) poly(PEGMEMA $\left.25 / \mathrm{STFLi}_{15}\right)$, poly(EHA $\left.{ }_{34} / \mathrm{STFLi}_{6}\right)$ and poly $\left(\mathrm{PEGMEMA}_{25} / \quad \mathrm{STFLi}_{15}\right)$ and (B) $\operatorname{poly}\left(\mathrm{GCA}_{25} / \mathrm{STFLi}_{15}\right), \quad \operatorname{poly}\left(\mathrm{GCA}_{30} / \mathrm{STFLi}_{10}\right)$, poly $\left(\mathrm{DTTA}_{20} / \mathrm{STFLi}_{20}\right)$ and poly$\left(\mathrm{MSEA}_{21} / \mathrm{STFLi}_{19}\right)$. 

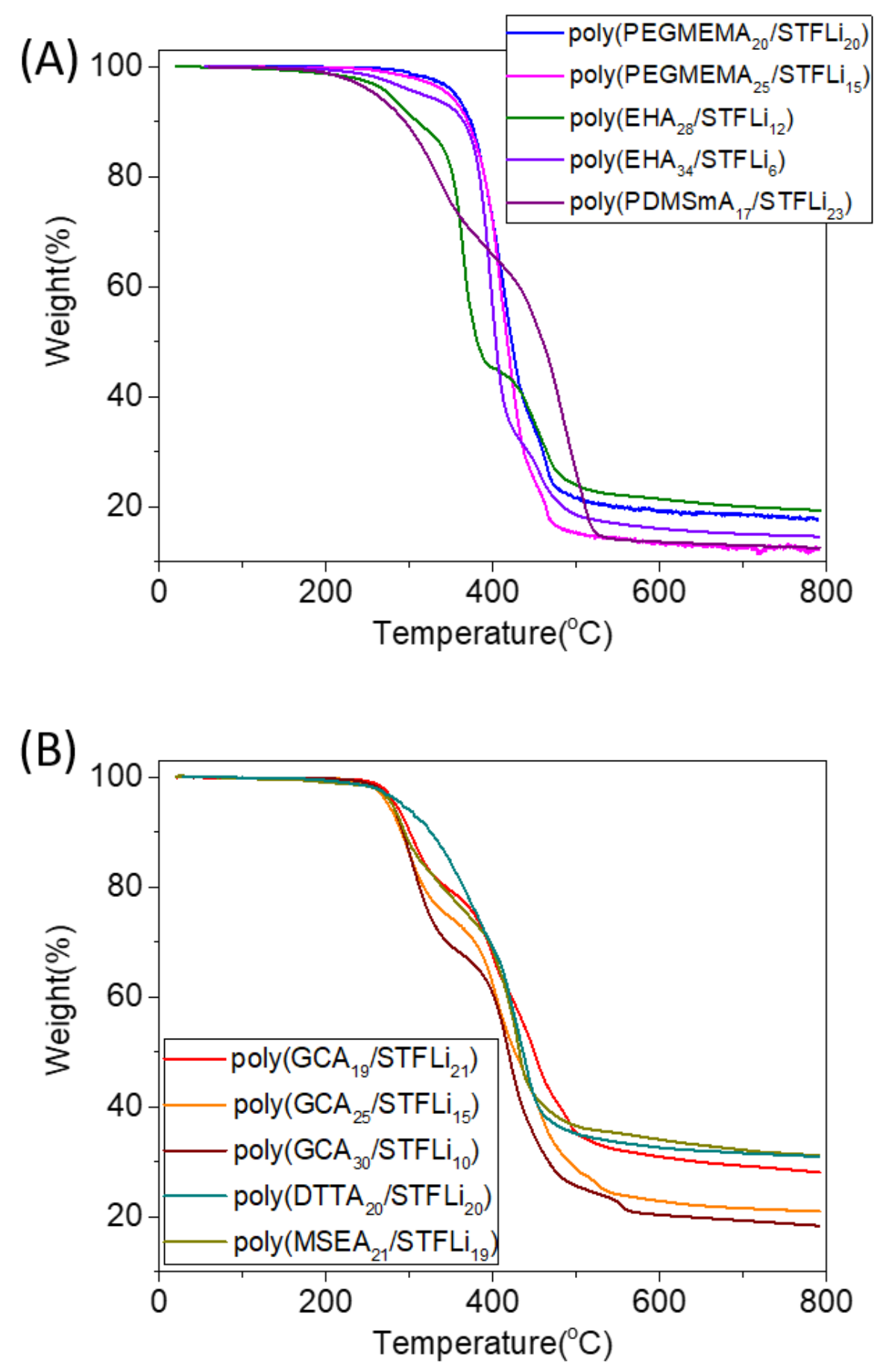

Figure S11. TGA spectra of all SICPEs. 


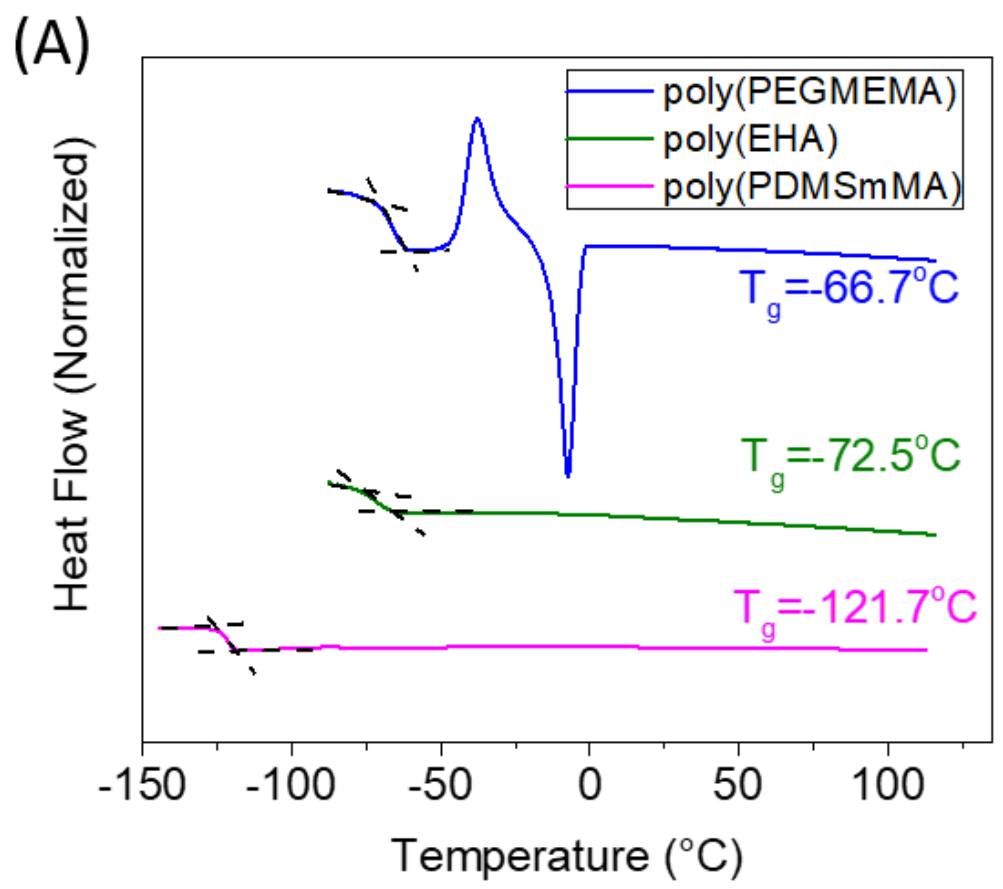

(B)

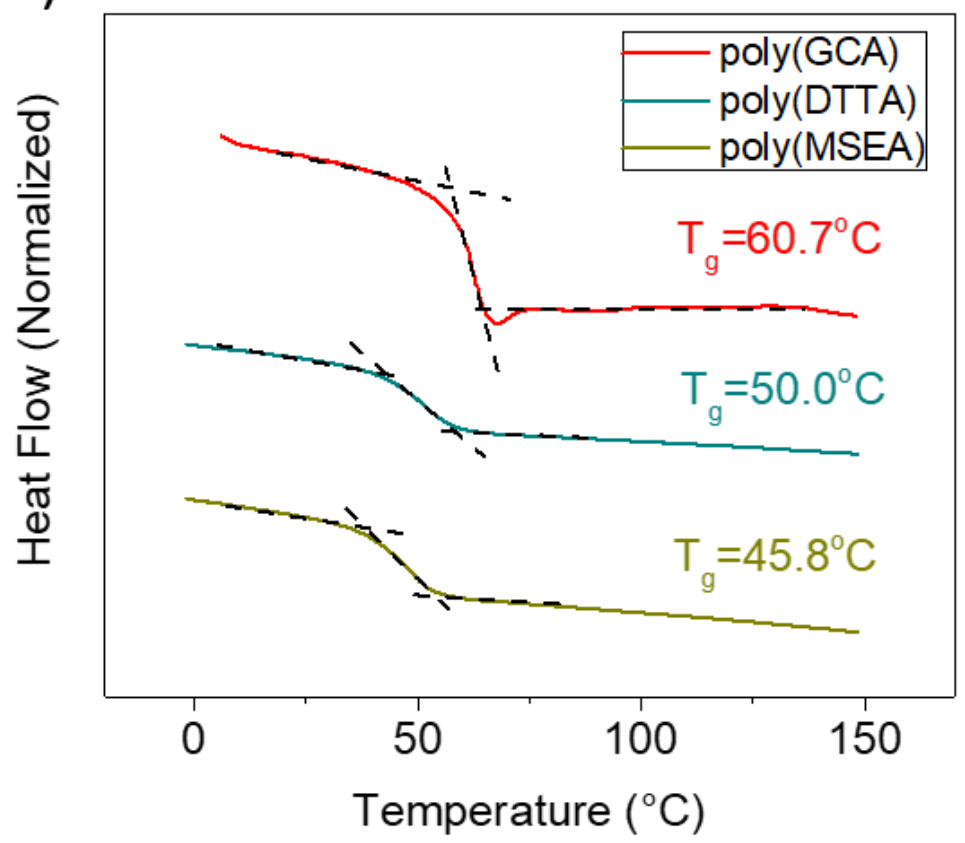

Figure S12. DSC curves for homopolymer. 
(A)

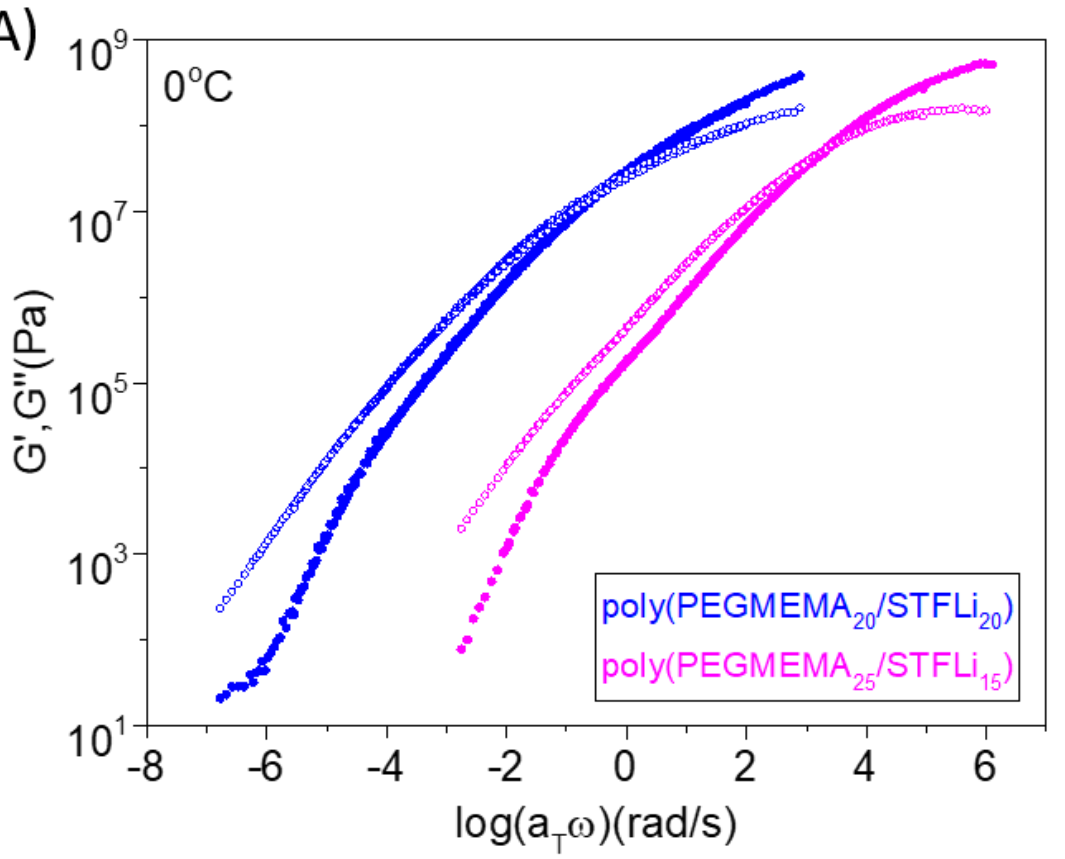

(B)

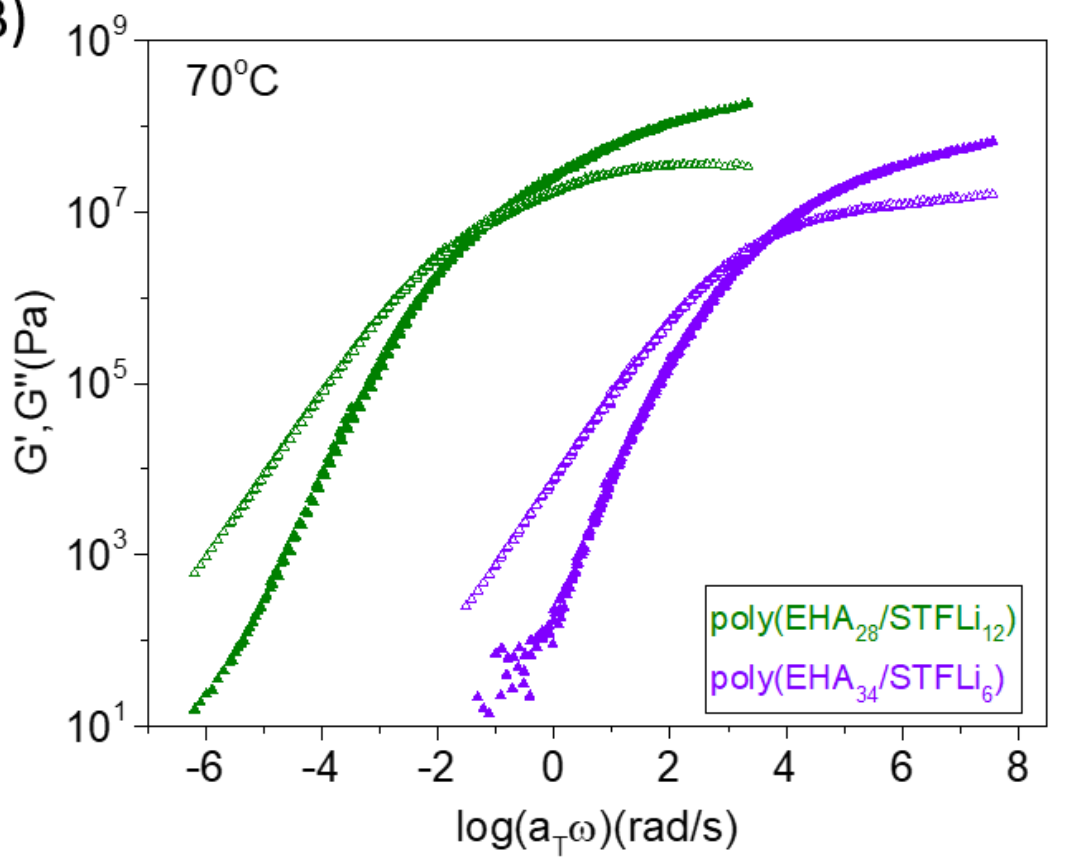

Figure S13. Modulus Master-curve of poly(PEGMEMA $\left./ \mathrm{STFLi}_{\mathrm{y}}\right)$ and poly $\left(\mathrm{EHA}_{\mathrm{x}} / \mathrm{STFLi}_{\mathrm{y}}\right)$. 


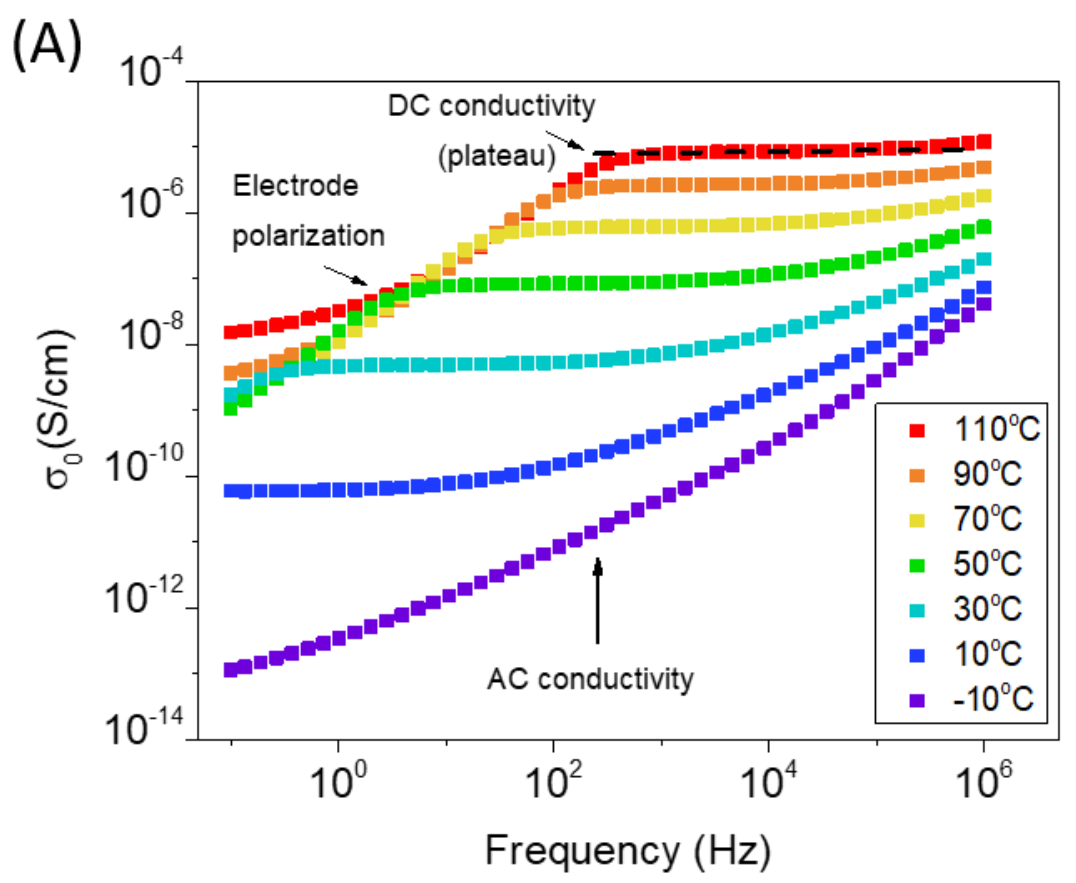

(B)

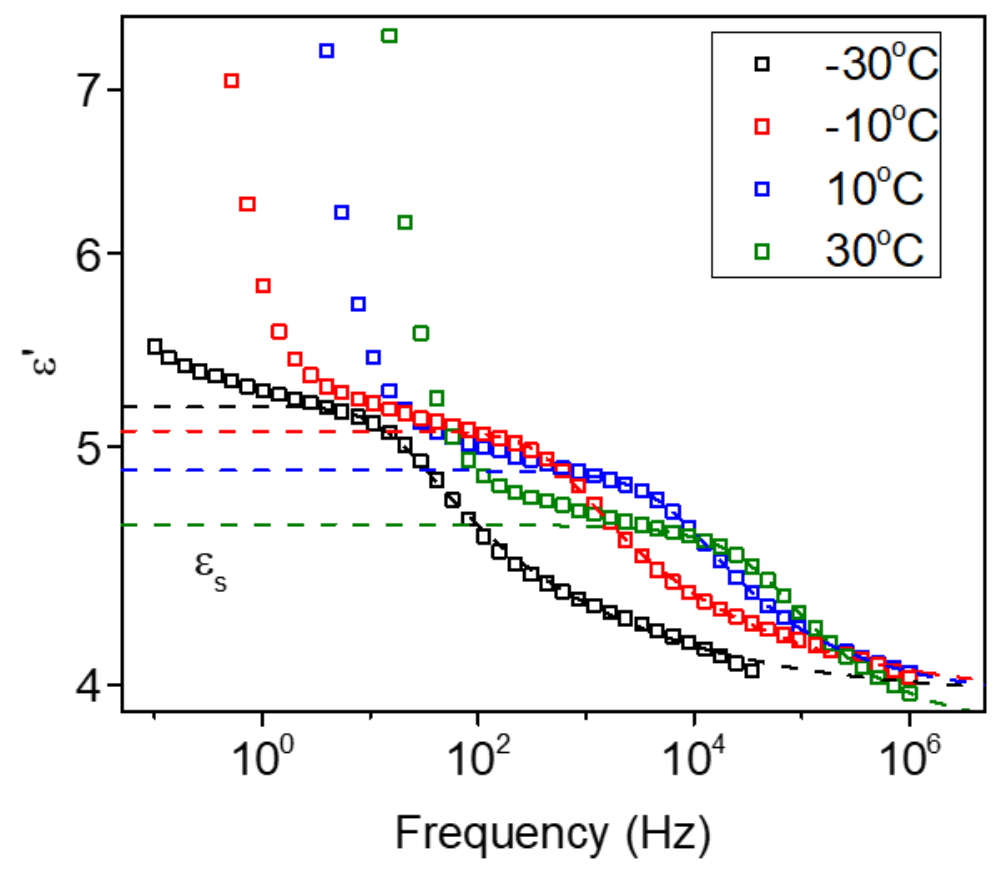

Figure S14. (A) Real part of conductivity spectrum for poly(PEGMEMA $20 / \mathrm{STFLi}_{20}$ ) and (B) real part of permittivity spectrum for poly(PEGMEMA). The permittivity spectra are fitted by HN function without electrode polarization (dashed-line). 


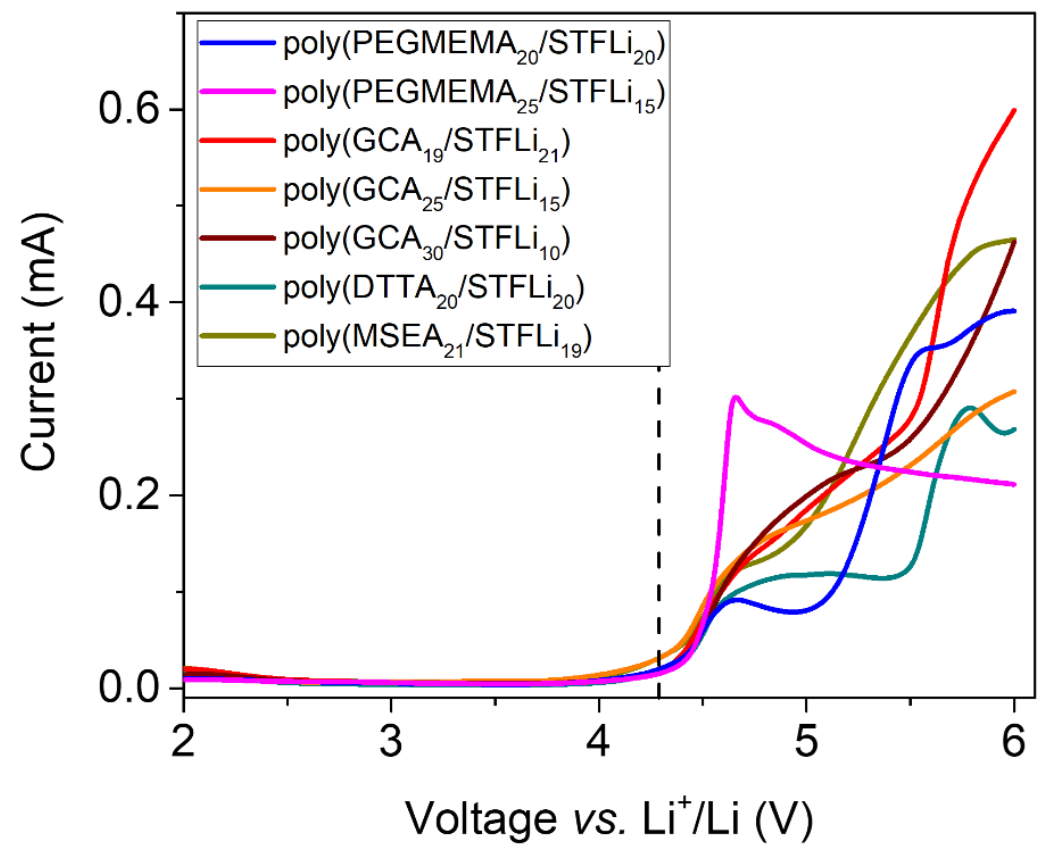

Figure S15. Linear sweep voltammetry (LSV) results of $10 \mathrm{wt} \%$ selected SICPEs doped in propylene carbonate (PC). 


\section{THEORETICAL SIMULATION}

\section{Computational Details}

Similar to our previous work, ${ }^{5}$ the OPLS-AA force field was used to investigate the non-covalent interactions for the current complex systems. ${ }^{6}$ The OPLS-AA force field has been extensively evaluated in chemical systems and has been proved to be reliable. ${ }^{7,8}$ The molecule specific parameter sets for PEGMEMA, PDMSmMA, EHA, GCA, and STF were generated by LigParGen

server. ${ }^{9,}{ }^{10}$ All the simulations were performed with the GROMACS 2018 software package. ${ }^{11,12}$ Four systems, i.e., PEGMEMA-STFLi, PDMSmMA-STFLi, EHA-STFLi, and GCA-STFLi, were constructed with ratios $1: 1,1: 1,3: 1$ and $3: 1$, respectively. A representative structure of each molecule is shown in Figure S16. When establishing the initial configuration of the simulation systems, the initial coordinates of each molecule were generated randomly. The convergence criterion of energy minimization was $1000 \mathrm{~kJ} \mathrm{~mol}^{-1} \mathrm{~nm}^{-1}$, and NVT ensembles at $300 \mathrm{~K}$ were performed separately. In production simulations, the velocity rescaling thermostat with a time constant of 0.1 ps was selected as the temperature coupling method. ${ }^{13}$ The Berendsen pressure coupling with a time constant of 1.0 ps was selected as the pressure coupling method, and the isothermal compression factor was set to $4.5 \times 10^{-5} \mathrm{bar}^{-1} .{ }^{14}$ The time step was $2 \mathrm{fs}$, and the periodic boundary conditions were applied in all directions of the simulated box. ${ }^{15}$ Bond lengths were constrained by the LINCS algorithm. ${ }^{16}$ During the simulation, the cut-off distance of van der Waals interactions was set at $1.2 \mathrm{~nm}$, and Coulombic interaction used the particle-mesh Ewald (PME) summation method. ${ }^{17,18}$ Figures of molecular structures in the computational sections are prepared using Chimera. ${ }^{19}$ 
The binding energy was calculated based on the largest cluster (identified from cluster analysis) by first-principle DFT calculations using the Vienna Ab-Initio Simulation Package (VASP). ${ }^{20,21}$ The exchange-correction interaction was described by the generalized gradient approximation parametrized by Perdew-Burke-Ernzerhof (PBE) functional. ${ }^{22}$ The projected augmented wave (PAW) method was applied. ${ }^{23,24}$ The valence electrons were described by plane-wave basis sets with an energy cutoff of $400 \mathrm{eV}$.

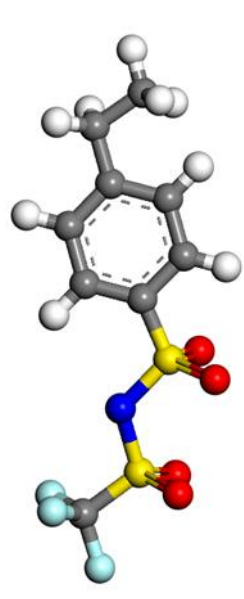

STF
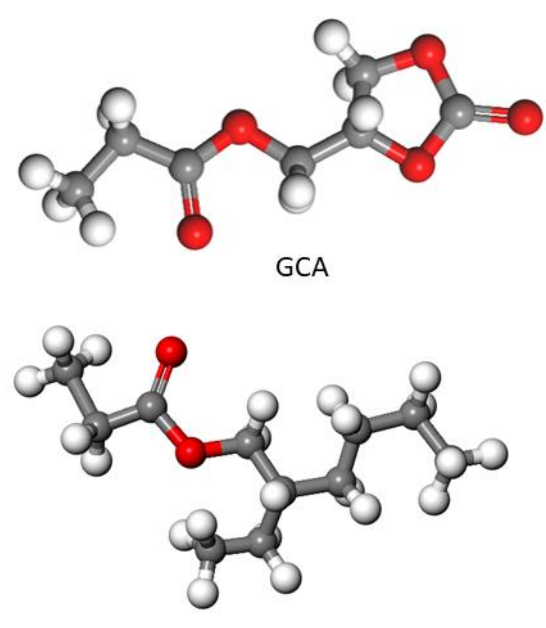

EHA

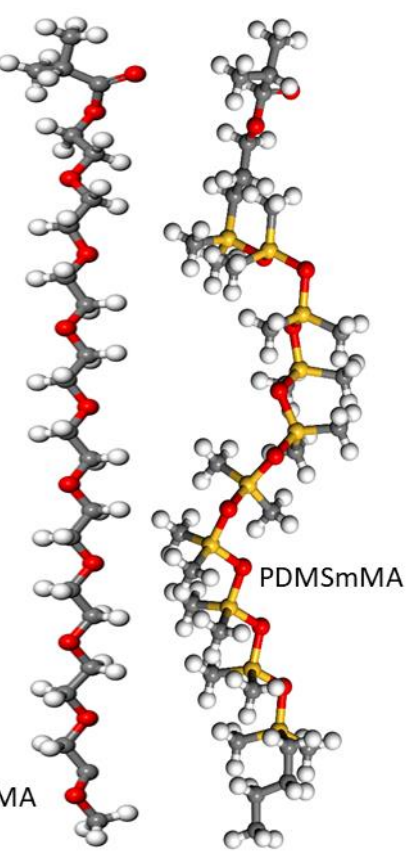

Figure S16. Ball and stick view of each molecule used in simulations.

\section{Interaction between $\mathrm{STF}^{-}, \mathrm{Li}^{+}$, and PEGMEMA}

Current simulations have revealed unique properties of the full-length PEGMEMA interacting with $\mathrm{Li}^{+}$. The results reveal a molecular interaction pattern in PEGMEMA-STFLi, where the two molecules form a ring-like structure with a lithium ion embedded at the core of the interaction between the two as shown in Figure S17. This structure is similar to crown ethers, where $\mathrm{Li}^{+}$bind with oxygen atoms within PEGMEMA through electrostatic interactions and the oxygen atoms 
protruding into the inner circle of the ring. ${ }^{25}$ Additional analysis using the radial distribution function (RDF) indicates that a large number of oxygen atoms in PEGMEMA are distributed at a position $2.5 \AA$ from lithium ions. In addition to the oxygen atoms, the nitrogen atom in $\mathrm{STF}^{-}$can also interact with $\mathrm{Li}^{+}$through the electrostatic interactions. Interestingly, this nitrogen atom in $\mathrm{STF}^{-}$is more negatively charge than the oxygen atoms in PEGMEMA. To identify the major contributor to electrostatic interactions among oxygen and nitrogen atoms to $\mathrm{Li}^{+}$, the radial distribution functions of oxygen and nitrogen atoms in $\mathrm{STF}^{-}$around $\mathrm{Li}^{+}$were investigated (shown in Figure S18(A)). The distribution peak corresponding to the nitrogen-lithium interaction is both much stronger and occurs at a shorter distance than that of oxygen atoms. This demonstrates that the nitrogen atoms in $\mathrm{STF}^{-}$are the major contributors to interactions with $\mathrm{Li}^{+}$.

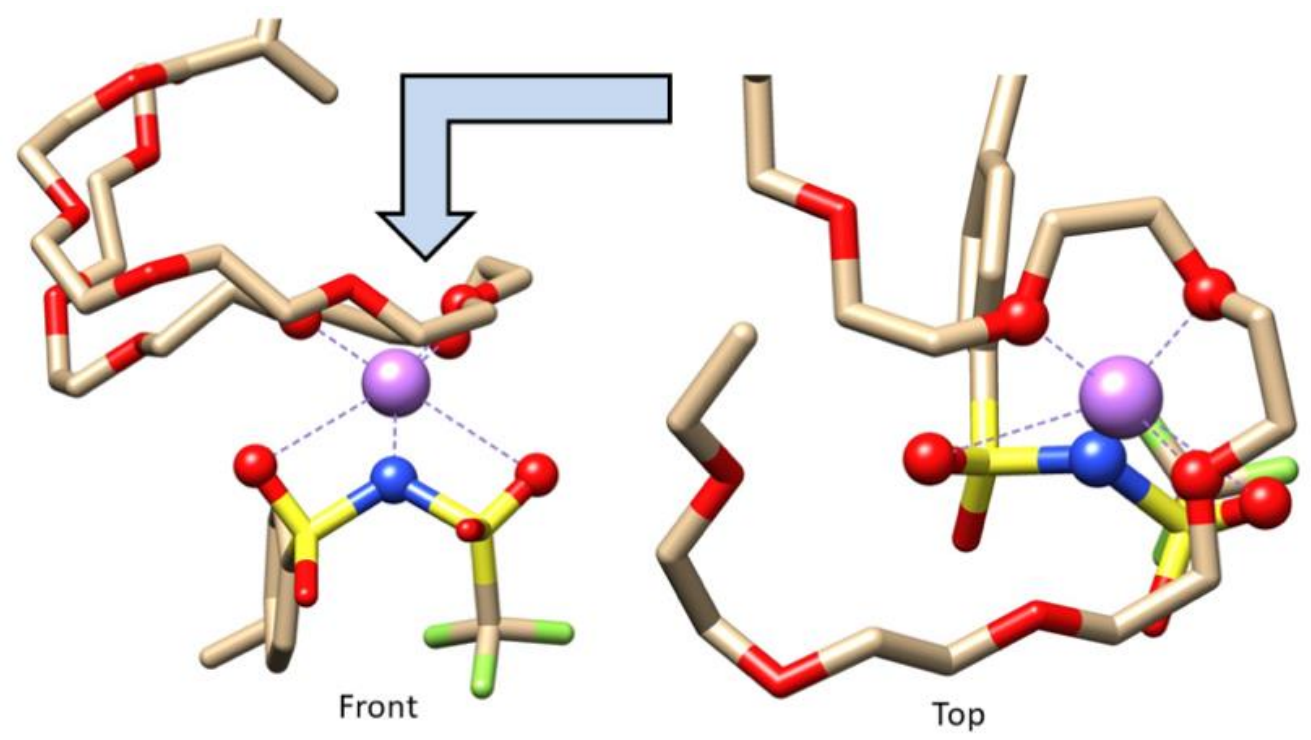

Figure S17. A representative interaction pattern of PEGMEMA-STFLi complex. 


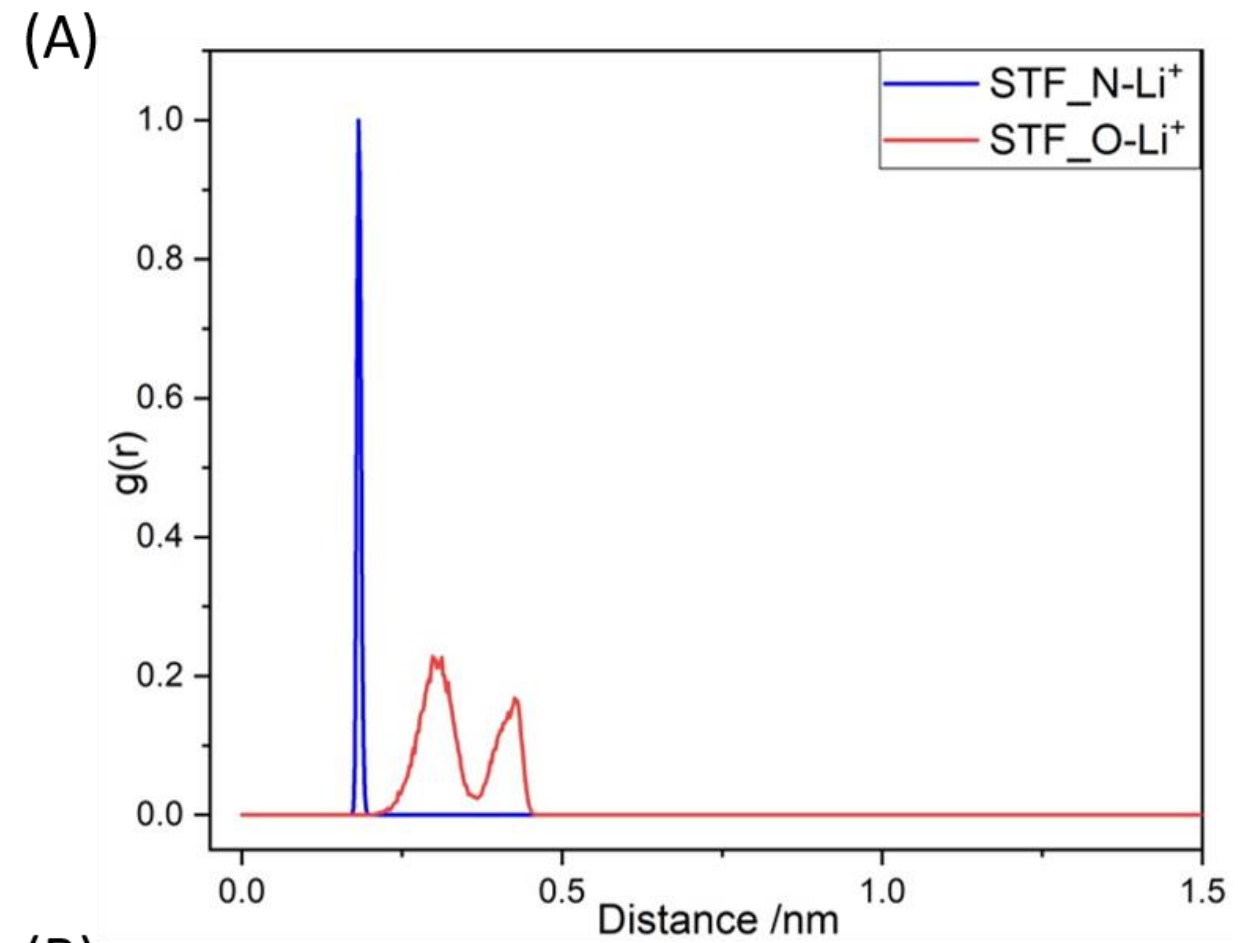

(B)

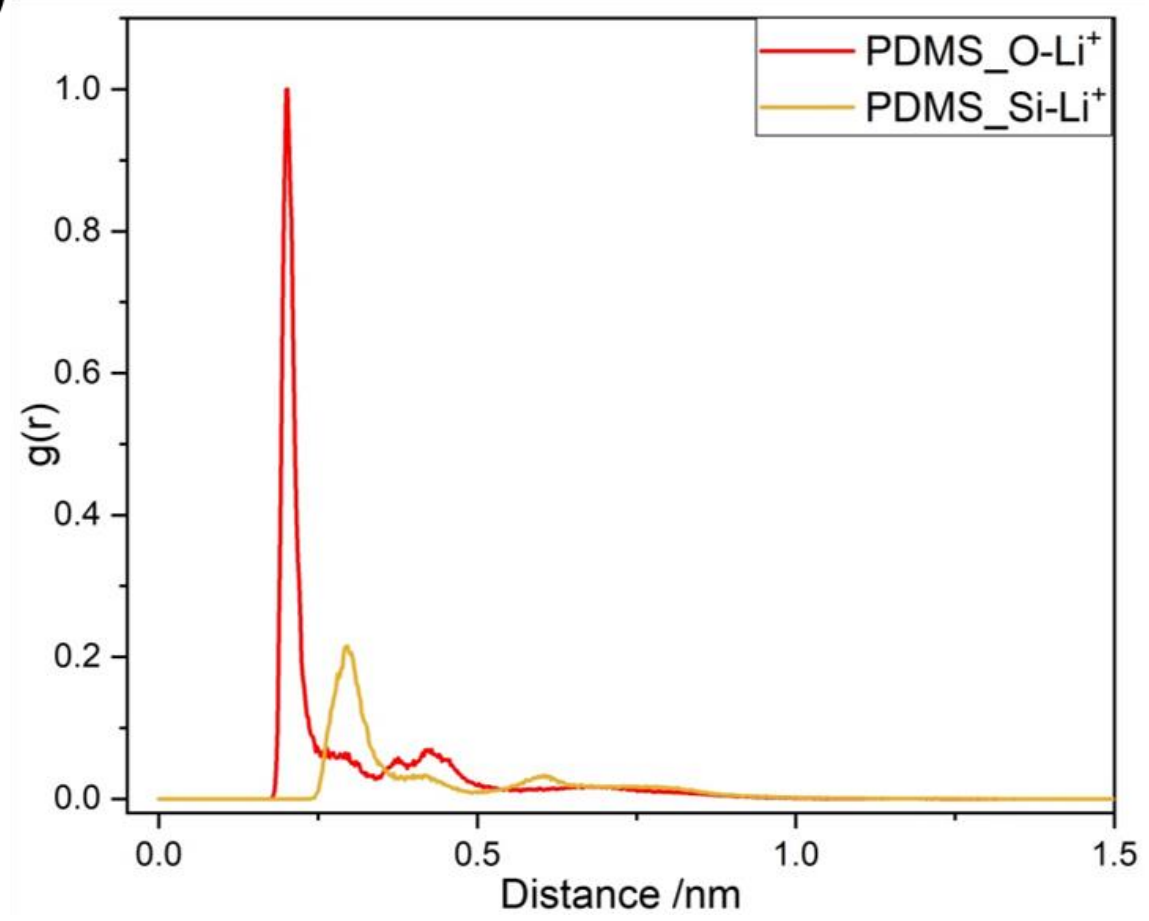

Figure S18. The radial distribution functions of (A) O and N atoms in STF and (B) O and Si atoms in PDMSmMA with respect to $\mathrm{Li}^{+}$. 


\section{Interaction between $\mathrm{STF}^{-}, \mathrm{Li}^{+}$, and PDMSmMA}

To study the interaction between PDMSmMA and STFLi, the molecular system containing fulllength PDMSmMA monomer and STFLi was built with a ratio of 1:1., As the silicon atoms in PDMSmMA monomer have a strong electrostatic repulsive interaction with lithium ions, their presence allows only a few oxygen atoms to form electrostatic attraction interactions with lithium ions.

The RDF characterizes the distributions of oxygen and silicon atoms in PDMS around lithium ions (Figure S18(B)). The distances from lithium ions to silicon atoms and oxygen atoms have different distribution peaks, and the peak of oxygen atoms is higher than that of silicon atoms. Such RDF results prove the idea that lithium ions interact with oxygen atoms in PDMS. However, most of the oxygen atoms in the side chain of PDMS are covalently bonded to two silicon atoms. Therefore, when oxygen atoms and lithium ions attract each other, silicon atoms are also located slightly further from lithium ions and are generating repelling forces to lithium ions. Such a combination of oxygen and silicon atoms could lead to the deformation of the complex.

\section{Interaction between $\mathrm{STF}^{-}, \mathrm{Li}^{+}$, EHA, and GCA}

In EHA-STFLi system, notably, there are only two oxygen atoms from ester group that may interact with lithium ions. As these oxygen atoms are close to the carbon backbone in EHA, they are less likely to interact with lithium ions due to the localized structure. Thus, compared with PEGMEMA, the interaction between EHA and $\mathrm{Li}^{+}$is weaker.

Different from EHA, GCA molecules also contain carbonyl oxygen atoms, which are more suitable to forms stronger interactions with $\mathrm{Li}^{+}$. However, this interaction is weaker compared to 
PEGMEMA with lithium ions. Furthermore, each GCA molecule can potentially interact with multiple $\mathrm{Li}^{+}$at the same time for a system containing multiple $\mathrm{Li}^{+}$and multiple $\mathrm{STF}^{-}$molecules.

\section{Calculation of Binding Energy}

To obtain the binding energy between different types of monomers and STFLi, the binding energy of the $\mathrm{Li}^{+}$, $\mathrm{STF}^{-}$, and a polymeric unit (PEGMEMA, PDMSmMA, GCA, EHA) was calculated by first-principle DFT methods. Detailed information of the DFT calculations can be found in the section titled "Computational Details". The binding energy estimation functions are the same as published in Cao et al. ${ }^{1}$ The binding energy of each complex is shown in Table S1. Table S1. Binding Energy of each complex.

\begin{tabular}{|l|l|l|l|l|}
\hline & EPEGMEMA+STF+Li & EGCA+STF+Li $_{\text {GeHA+STF+Li }}$ & EPDMS+STF+Li \\
\hline Binding Energy & $-0.47063 \mathrm{ev}$ & $-0.27012 \mathrm{ev}$ & $-0.22373 \mathrm{ev}$ & $3.038214 \mathrm{ev}$ \\
\hline
\end{tabular}

The calculated binding energies suggest that the PEGMEMA has the lowest binding energy, which indicates PEGMEMA+STFLi to be the most stable complex among all four complexes listed above. The stability of complex with STFLi is followed by GCA, EHA, and PDMS, respectively, which agree with the experimental measurements and the conclusion derived from contact analysis. Furthermore, the positive binding energy reported for the complex PDMS+STFLi suggests that the complex is not stable and prefer to deform. 


\section{REFERENCE}

1. Lim, J.; Yang, H.; Paek, K.; Cho, C.-H.; Kim, S.; Bang, J.; Kim, B. J. “Click” Synthesis of Thermally Stable Au Nanoparticles with Highly Grafted Polymer Shell and Control of Their Behavior in Polymer Matrix. J. Polym. Sci., Part A: Polym. Chem. 2011, 49 (16), 3464-3474.

2. Ye, P.; Cao, P.-F.; Su, Z.; Advincula, R. Highly Efficient Reversible AdditionFragmentation Chain-Transfer Polymerization in Ethanol/Water via Flow Chemistry. Polym. Int. 2017, $66(9), 1252-1258$.

3. Cao, P.-F.; Wojnarowska, Z.; Hong, T.; Carroll, B.; Li, B.; Feng, H.; Parsons, L.; Wang, W.; Lokitz, B. S.; Cheng, S.; Bocharova, V.; Sokolov, A. P.; Saito, T. A Star-Shaped Single Lithium-Ion Conducting Copolymer by Frafting a POSS Nanoparticle. Polymer 2017, 124, $117-127$.

4. Meziane, R.; Bonnet, J.-P.; Courty, M.; Djellab, K.; Armand, M. Single-Ion Polymer Electrolytes based on a Delocalized Polyanion for Lithium Batteries. Electrochim. Acta 2011, 57, $14-19$.

5. Cao, P.-F.; Li, B.; Yang, G.; Zhao, S.; Townsend, J.; Xing, K.; Qiang, Z.; Vogiatzis, K. D.; Sokolov, A. P.; Nanda, J.; Saito, T. Elastic Single-Ion Conducting Polymer Electrolytes: Toward a Versatile Approach for Intrinsically Stretchable Functional Polymers. Macromolecules 2020, 53 (9), 3591-3601.

6. Jorgensen, W. L.; Maxwell, D. S.; Tirado-Rives, J. Development and Testing of the OPLS All-Atom Force Field on Conformational Energetics and Properties of Organic Liquids. J. Am. Chem. Soc. 1996, 118, 11225-11236. 
7. Muralidharan, A.; Chaudhari, M. I.; Pratt, L. R.; Rempe, S. B. Molecular Dynamics of Lithium Ion Transport in a Model Solid Electrolyte Interphase. Sci. Rep. 2018, 8 (1), 10736.

8. Shim, Y. Computer Simulation Study of the Solvation of Lithium Ions in Ternary Mixed Carbonate Electrolytes: Free Energetics, Dynamics, and Ion Transport. Phys. Chem. Chem. Phys. 2018, 20 (45), 28649-28657.

9. Jorgensen, W. L.; Tirado-Rives, J. Potential Energy Functions for Atomic-Level Simulations of Water and Organic and Biomolecular Systems. Proc. Natl. Acad. Sci. U. S. A. 2005, $102(19), 6665-6670$.

10. Dodda, L. S.; Vilseck, J. Z.; Tirado-Rives, J.; Jorgensen, W. L. 1.14*CM1A-LBCC: Localized Bond-Charge Corrected CM1A Charges for Condensed-Phase Simulations. J. Phys. Chem. C 2017, 121 (15), 3864-3870.

11. Berendsen, H. J. C.; Spoel, D. v. d.; Drunen, R. v. GROMACS: A Message-Passing Parallel Molecular Dynamics Implementation. Comput. Phys. Commun. 1995, 91 (1-3), 43-56.

12. Abraham, M. J.; Murtola, T.; Schulz, R.; Páll, S.; Smith, J. C.; Hess, B.; Lindahl, E. GROMACS: High Performance Molecular Simulations through Multi-Level Parallelism from Laptops to Supercomputers. SoftwareX 2015, 1-2, 19-25.

13. Bussi, G.; Donadio, D.; Parrinello, M. Canonical Sampling through Velocity Rescaling. $J$. Chem. Phys. 2007, $126(1), 014101$.

14. Berendsen, H. J. C.; Postma, J. P. M.; van Gunsteren, W. F.; DiNola, A.; Haak, J. R. Molecular Dynamics with Coupling to an External Bath. J. Chem. Phys. 1984, 81 (8), 3684-3690. 
15. Apostolakis, J.; Ferrara, P.; Caflischa, A. Calculation of Conformational Transitions and Barriers in Solvated Systems Application to the Alanine Dipeptide in Water. J. Chem. Phys. 1999, $110(4), 2099-2108$.

16. Hess, B.; Bekker, H.; Beerndsen, H. J. C.; JFraaije, o. G. E. M. LINCS: A Linear Constraint Solver for Molecular Simulations. J. Comput. Chem. 1997, 18 (12), 1463-1472.

17. Essmann, U.; Perera, L.; Berkowitz, M. L.; Darden, T.; Lee, H.; Pedersen, L. G. A Smooth Particle Mesh Ewald Method. J. Chem. Phys. 1995, 103 (19), 8577-8593.

18. Tom, D.; Darrin, Y.; Lee, P. Particle Mesh Ewald: An N· $\log (\mathrm{N})$ Method for Ewald Sums in Large Systems. J. Chem. Phys. 1993, 98 (12), 10089-10092.

19. Pettersen, E. F.; Goddard, T. D.; Huang, C. C.; Couch, G. S.; Greenblatt, D. M.; Meng, E. C.; Ferrin, T. E. UCSF Chimera--A Visualization System for Exploratory Research and Analysis. J. Comput. Chem. 2004, 25 (13), 1605-1612.

20. Kresse, G.; Furthmiiller, J. Efficiency of Ab-Initio Total Energy Calculations for Metals and Semiconductors using a Plane-Wave Basis Set. Comput. Mater. Sci. 1996, 6, 15-50.

21. Ishikawa, A.; Doi, T.; Nakai, H. Catalytic Performance of Ru, Os, and Rh Nanoparticles for Ammonia Synthesis: A Density Functional Theory Analysis. J. Catal. 2018, 357, 213-222.

22. Perdew, J. P.; Burke, K.; Ernzerhof, M. Generalized Gradient Approximation Made Simple. Phys. Rev. Lett. 1996, 77 (18), 3865-3868.

23. Blochl, P. E. Projector Augmented-Wave Method. Phys. Rev. B: Condens. Matter Mater. Phys. 1994, 50 (24), 17953-17979. 
24. Kresse, G.; Jouber, D. From Ultrasoft Pseudopotentials to the Projector Augmented-Wave Method. Phys. Rev. B: Condens. Matter Mater. Phys. 1999, 59 (3), 1758-1775.

25. Power, P. P. Free Inorganic, Organic, and Organometallic Ions by Treatment of Their Lithium Salts with 12-Crown-4. Acc. Chem. Res. 1988, 21, 147-153. 